\title{
Checklist of Central and South American Agaricales (Basidiomycota) II: Strophariaceae
}

\section{Coimbra VRM ${ }^{1}$}

${ }^{1}$ Universidade Federal de Pernambuco, Departamento de Micologia/CCB, Programa de Pós-graduação em Biologia de Fungos, Av. Prof. Nelson Chaves, s/n, CEP: 50670-901, Recife-PE, Brazil; Email: vick_mat_coimbra@yahoo.com.br

Coimbra VRM 2015 - Checklist of Central and South American Agaricales (Basidiomycota) II: Strophariaceae. Mycosphere 6(4), 441-458, Doi 10.5943/mycosphere/6/4/6

\begin{abstract}
Following a serie of papers inventorying the agaric mycota (Agaricales) of Central and South Americas, this second publication is a literature-based checklist of the dark-spored family Strophariaceae. In total, 288 taxa belonging to 18 genera are reported here, mainly for Argentina (120 spp.) and Brazil (115 spp.), the most representative countries. This list is an effort to organize the widespread knowledge of Strophariaceae species diversity and geographical distribution, but does not reflect the real diversity of Strophariaceae from this region.
\end{abstract}

Key words - agarics - America - diversity - Neotropics

\section{Introduction}

This paper is the second part of a series of extensive checklists inventorying the agaric mycota from Central and South Americas. Each annual volume has been dedicated to one of the most important families of agaricoid fungi (Agaricales), as for example, the Entolomataceae Kotl. \& Pouzar (Coimbra 2014).

This current volume deals with Strophariaceae Singer \& A.H. Smith, a large family of darkspored mushrooms with 1316 known species (Kirk et al. 2008), and distributed worldwide, since the Arctic to the Antarctic zones (Pegler et al. 1980, Singer 1986, Borgen et al. 2006).

The aim of this work is to provide a complete literature-based checklist of the Strophariaceae species recorded to Central and South America, as a tool for future studies on diversity, geographical distribution and species estimation.

\section{Methods}

The methodology of gathering data adopted here is the same of Coimbra (2014), as a way of standardization for all volumes of this series. Thus, only data recovered up to 10 March 2015 and published in papers and books were considered. Also, unidentified species (e.g. Pholiota sp.) and voucher numbers were not included. A special section ("Excluded species") was designated in the end of the list, for taxa names absent on the adopted databases (Mycobank - www.mycobank.org and Index Fungorum - www.indexfungorum.org) and for species referred as "excluded" in the literature (invalid names, "nomen dubium", species with lost types, etc).

Strophariaceae is recognised here, mainly, in the sense of Singer (1986), but also considering the recent advances on the understanding of this family (Matheny et al. 2014). Naematoloma P. Karst, despite of its illegitimacy (according to Mycobank), is maintened here 
because the two species known for that geographical region and belonging to this genus ( $N$. amazonicum Singer and N. nudum Singer) still needs proper combinations. Thus, the adopted genera in this checklist are: Agrocybe Fayod, Crassisporium Matheny, P.-A. Moreau \& Vizzini, Deconica (W.G. Sm.) P. Karst., Flammula (Schaeff.: Fr.) P. Kumm., Geophila Quél., Hypholoma (Fr.) P. Kumm., Kuehneromyces Singer \& A.H. Sm., Leratiomyces Bresinsky \& Manfr. Binder ex Bridge, Spooner, Beever \& D.C. Park, Melanotus Pat., Naematoloma, Naucoria (Fr.) P. Kumm., Pachylepyrium Singer, Phaeogalera Kühner, Pholiota (Fr.) P. Kumm., Protostropharia Redhead, Moncalvo \& Vilgalys, Psilocybe (Fr.) P. Kumm., Stagnicola Redhead \& A.H. Sm. and Stropharia (Fr.) Quél.

\section{Results and Discussion}

In total, 288 Strophariaceae taxa (281 species and 7 varities), belonging to 18 genera, were listed to Central and South Americas (Table 1) based on previously published data. These data were recovered from 113 publications (some with limited availabity), written in several languages (Danish, English, Latin, German, Portuguese and Spanish).

Among all genera, the neurotropic Psilocybe (Fr.) P. Kumm. was the most reported (Figure 1), mainly due to the extensive studies of the renowned specialist Gastón Guzmán and colleagues (Guzmán 1978, 1983, 1995, 2004, 2012, Guzmán \& Cortez 2005, Guzmán et al. 1984, 1994, 1998, 2003, 2004a, 2004b).

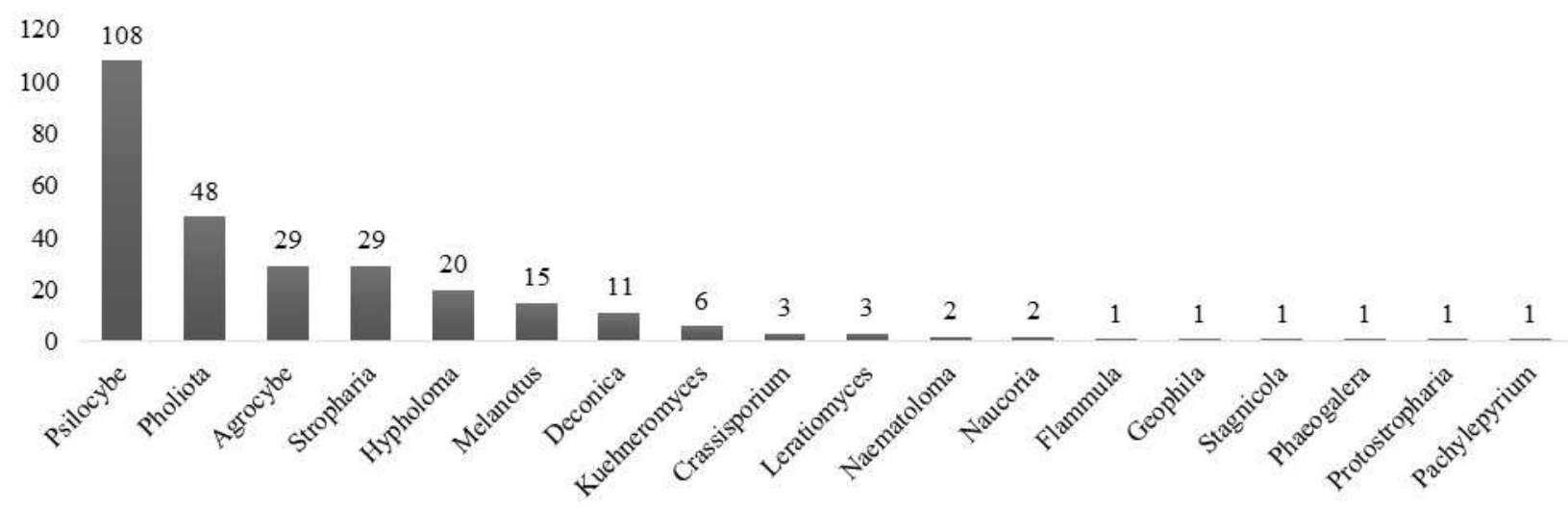

Fig. 1 - Number of Strophariaceae species for each genus, reported for Central and South America.

Table 1 List of Strophariaceae taxa recorded to Central and South America.

\begin{tabular}{|c|c|c|}
\hline Taxa & Distribution & Reference \\
\hline Agrocybe allocystis Singer & $\begin{array}{l}\text { ARG, BRA, CHI, } \\
\text { URU }\end{array}$ & $\begin{array}{l}\text { Singer (1969), Wright \& Albertó (2002) and } \\
\text { Meijer (2006) }\end{array}$ \\
\hline Agrocybe arenicola (Berk.) Singer & ARG & Wright \& Albertó (2002) \\
\hline Agrocybe broadwayi (Murrill) Dennis & $\begin{array}{l}\text { BRA, CUB, GRN, } \\
\text { GUF, TTO }\end{array}$ & $\begin{array}{l}\text { Pegler (1983, 1997) and Courtecuisse (1996); } \\
\text { Pegler (1987a) as Agaricus semiorbicularis }\end{array}$ \\
\hline Agrocybe collybiiformis (Murrill) Singer & BRA & Pegler $(1997)$ \\
\hline Agrocybe coprophila (Rick) Singer & ARG, BRA & $\begin{array}{l}\text { Wright \& Albertó (2002); Rick }(1938,1961) \\
\text { as Hebeloma coprophilum. }\end{array}$ \\
\hline Agrocybe cubensis (Murrill) Singer & BRA, CUB & $\begin{array}{l}\text { Pegler (1987b), Watling (1992) and Meijer } \\
\text { (2006) }\end{array}$ \\
\hline Agrocybe cylindracea (DC.: Fr.) Maire & $\begin{array}{l}\text { ARG, BRA, CHI, } \\
\text { GUA }\end{array}$ & $\begin{array}{l}\text { Watling (1992), Pegler (1997), Wright \& } \\
\text { Albertó (2002), Meijer (2006) and George- } \\
\text { Nascimento (2007); Spegazzini (1899) and } \\
\text { Rick (1938, 1961) as Pholiota impudica; Boa } \\
\text { (2004) and Flores Arzú et al. (2012) as } \\
\text { Agrocybe aegerita. }\end{array}$ \\
\hline Agrocybe earlei (Murrill) Dennis & $\begin{array}{l}\text { BRA(?), } \quad \text { COL, } \\
\text { JAM, MTQ }\end{array}$ & $\begin{array}{l}\text { Pegler (1983), Watling (1992), Meijer (2006) } \\
\text { and Franco-Molano et al. (2010) }\end{array}$ \\
\hline
\end{tabular}




\begin{tabular}{|c|c|c|}
\hline Taxa & Distribution & Reference \\
\hline Agrocybe fimicola (Speg.) Singer & ARG, BRA & $\begin{array}{l}\text { Watling (1992), Wright \& Albertó (2002) and } \\
\text { Meijer (2006); Rick (1938, 1961) as Naucoria } \\
\text { pediades. }\end{array}$ \\
\hline Agrocybe lazoi Singer & $\mathrm{CHI}$ & Singer (1969) \\
\hline Agrocybe lenticeps (Peck) Singer & $\mathrm{ARG}$ & Singer (1973) \\
\hline Agrocybe musae (Earle) Pegler & CUB & Pegler (1987b) \\
\hline Agrocybe neocoprophila Singer & BRA & $\begin{array}{l}\text { Singer }(1953,1965) \text {; Rick }(1938,1961) \text { as } \\
\text { Hebeloma coprophilum. }\end{array}$ \\
\hline Agrocybe paradoxa Singer & $\mathrm{ARG}$ & Singer $(1973)$ \\
\hline Agrocybe pediades (Fr.) Fayod & $\mathrm{ARG}$ & $\begin{array}{l}\text { Wright \& Albertó (2002); Singer (1969) also } \\
\text { as A. semiorbicularis }\end{array}$ \\
\hline Agrocybe perfecta (Rick) Singer & BRA & $\begin{array}{l}\text { Singer (1953), Watling (1992) and Meijer } \\
\text { (2006); Rick (1938, 1961) as Pholiota } \\
\text { vermiflua. }\end{array}$ \\
\hline Agrocybe platensis (Speg.) Singer & ARG, CHI & Singer (1969) \\
\hline Agrocybe praecox (Pers.) Fayod & $\begin{array}{l}\text { ARG, BRA, CHI, } \\
\text { COL, VEN }\end{array}$ & $\begin{array}{l}\text { Dennis (1961), Singer (1969), Franco-Molano } \\
\& \text { Uribe-Calle (2000) and Delgado \& } \\
\text { Urdaneta (2002); Rick (1938, 1961) as } \\
\text { Pholiotella gibberosa. }\end{array}$ \\
\hline Agrocybe procera Singer & ARG, $\mathrm{CHI}$ & Singer (1969) \\
\hline Agrocybe puiggarii (Speg.) Singer & BRA & $\begin{array}{l}\text { Pegler (1997) and Meijer (2006); Spegazzini } \\
\text { (1899) as Pholiota puiggarii; Rick (1938, } \\
\text { 1961) as P. praecox. }\end{array}$ \\
\hline Agrocybe retigera (Speg.) Singer & $\begin{array}{l}\text { ARG, BRA, PAR, } \\
\text { VEN }\end{array}$ & $\begin{array}{l}\text { Singer \& Digilio (1951), Delgado \& Urdaneta } \\
\text { (2002), Wright \& Albertó (2002) and Cortez } \\
\& \text { Silveira (2005); Spegazzini (1922) as } \\
\text { Naucoria retigera }\end{array}$ \\
\hline Agrocybe sacchari (Murrill) Dennis & BRA(?), VEN & Dennis (1961) and Grandi et al. (1984) \\
\hline Agrocybe sororia (Peck) Watling & BRA & Watling (1992) and Meijer (2006) \\
\hline Agrocybe subpediades (Murrill) Watling & BRA & Watling (1992) and Meijer (2006) \\
\hline Agrocybe tropicalis (Speg.) Guzmán & BRA & Pegler (1997) \\
\hline Agrocybe tucumana (Singer) Watling & ARG, BRA & $\begin{array}{l}\text { Singer (1973), Pegler (1997) and Meijer } \\
(2006)\end{array}$ \\
\hline Agrocybe underwoodii (Murrill) Singer & BRA, CUB & $\begin{array}{l}\text { Watling (1992) and Meijer (2006); Pegler } \\
\text { (1987b) as Naucoria underwoodii }\end{array}$ \\
\hline Agrocybe vervacti (Fr.) Singer & BRA & Rick $(1930,1938,1961)$ as Naucoria vervacti \\
\hline Agrocybe viscosa Singer & $\mathrm{CHI}$ & Singer (1969) \\
\hline $\begin{array}{l}\text { Crassisporium chilense (M.M. Moser) } \\
\text { Matheny, P.-A. Moreau \& Vizzini }\end{array}$ & $\mathrm{CHI}$ & Moser (2000) \\
\hline $\begin{array}{l}\text { Crassisporium funariophilum (M.M. Moser) } \\
\text { Matheny, P.-A. Moreau \& Vizzini }\end{array}$ & $\mathrm{CHI}$ & $\begin{array}{l}\text { Singer (1969) as Pachylepyrium } \\
\text { funariophilum }\end{array}$ \\
\hline $\begin{array}{l}\text { Crassisporium } \quad \text { squarrulosum } \\
\text { Matheny, P.-A. Moreau \& Vizzini }\end{array}$ & $\mathrm{CHI}$ & Singer (1969) as Pachylepyrium squarrulosum \\
\hline Deconica argentina Speg. & $\begin{array}{l}\text { ARG, BRA, COL, } \\
\text { PER, VEN }\end{array}$ & $\begin{array}{l}\text { Spegazzini (1899); Singer }(1969) \text {, } \\
\text { Raithelhuber }(1974,1988,1991, \text { 2004), } \\
\text { Guzmán (1978, 1995), Yokoyama (1987), } \\
\text { Franco-Molano \& Uribe-Calle (2000), Wright } \\
\text { \& Albertó (2002), Wartchow et al. (2007) and } \\
\begin{array}{l}\text { Niveiro \& Albertó (2012) as Psilocybe } \\
\text { argentina }\end{array}\end{array}$ \\
\hline Deconica coprophila (Bull.) P. Karst. & $\begin{array}{l}\text { ARG, BRA, COL, } \\
\text { ECU, GUF, PAN, } \\
\text { PER, VEN }\end{array}$ & $\begin{array}{l}\text { Silva et al. (2012); Spegazzini (1887b, 1899, } \\
\text { 1925, 1926b), Rick (1938, 1961), Dennis } \\
\text { (1961), Guzmán (1978, 1995), Horak (1980), } \\
\text { Yokoyama (1987), Stijve \& Meijer (1993), } \\
\text { Guzmán et al. (1994), Courtecuisse et al. } \\
\text { (1996), Cortez \& Coelho (2004), Meijer } \\
\text { (2006), Silva et al. (2006, 2008) and Niveiro } \\
\text { \& Albertó (2012) as Psilocybe coprophila }\end{array}$ \\
\hline Deconica horizontalis (Bull.) Noordel. & ARG, BRA & $\begin{array}{l}\text { Silva et al. (2012); Rick (1938, 1961) as } \\
\text { Melanotus hepatochrous; Cortez \& Coelho } \\
\text { (2004) as M. proteus; Niveiro \& Albertó } \\
\text { (2012) as M. horizontalis }\end{array}$ \\
\hline
\end{tabular}




\begin{tabular}{|c|c|c|}
\hline Taxa & Distribution & Reference \\
\hline Deconica merdaria (Fr.) Noordel. & $\begin{array}{l}\text { ARG, BRA, CHI, } \\
\text { URU, VEN }\end{array}$ & $\begin{array}{l}\text { Rick (1907, 1939, 1961) as Stropharia } \\
\text { merdaria; Guzmán (1978) and Valenzuela et } \\
\text { al. (1992) as Psilocybe merdaria }\end{array}$ \\
\hline Deconica moelleri (Guzmán) Noordel. & $\begin{array}{l}\text { ARG, BRA, CHI, } \\
\text { PER }\end{array}$ & $\begin{array}{l}\text { Spegazzini }(1899,1926 b) \text { as Stropharia } \\
\text { merdaria; Singer (1969) as Psilocybe } \\
\text { merdaria var. macrospora; Guzmán (1978), } \\
\text { Cortez \& Coelho (2004) and Niveiro \& } \\
\text { Albertó (2012) as P. moelleri }\end{array}$ \\
\hline Deconica montana (Pers.) P.D. Orton & CHI, COL, VEN & $\begin{array}{l}\text { Dennis (1961) as P. atrorufa; Singer (1969), } \\
\text { Guzmán (1978), Guzmán et al. (1994) and } \\
\text { Franco-Molano \& Uribe-Calle (2000) as } \\
\text { Psilocybe montana }\end{array}$ \\
\hline $\begin{array}{l}\text { Deconica musacearum (Singer) Cortez \& P.S. } \\
\text { Silva }\end{array}$ & BRA & $\begin{array}{l}\text { Silva et al. (2014); Singer (1989) and Meijer } \\
\text { (2006) as Melanotus musacearum }\end{array}$ \\
\hline $\begin{array}{l}\text { Deconica neorhombispora (Guzmán) P.S. } \\
\text { Silva, Ram.-Cruz \& Guzmán }\end{array}$ & BRA & $\begin{array}{l}\text { Silva et al. (2013); Silva et al. (2007) as } P \text {. } \\
\text { subbrunneocystidiata }\end{array}$ \\
\hline $\begin{array}{l}\text { Deconica pegleriana (Guzmán) Ram.-Cruz \& } \\
\text { Guzmán }\end{array}$ & $\begin{array}{l}\text { ARG, BRA, ECU, } \\
\text { VEN }\end{array}$ & $\begin{array}{l}\text { Guzmán et al. (1998), Moyano \& Daniele } \\
\text { (2003), Cortez \& Coelho (2004), Wartchow et } \\
\text { al. (2007) and Niveiro \& Albertó (2012) as } \\
\text { Psilocybe pegleriana }\end{array}$ \\
\hline Deconica phyllogena (Peck) Noordel. & COL, VEN & $\begin{array}{l}\text { Guzmán et al. (1994), Franco-Molano \& } \\
\text { Uribe-Calle (2000) and Franco-Molano et al. } \\
(2010) \text { as Psilocybe phyllogena; Dennis } \\
\text { (1961) as P. modesta }\end{array}$ \\
\hline Deconica subcoprophila (Britzelm.) E. Horak & ARG & $\begin{array}{l}\text { Singer (1969), Guzmán (1978), Horak (1980), } \\
\text { Raithelhuber (1988, 1991, 2004) and Niveiro } \\
\text { \& Albertó (2012) as Psilocybe subcoprophila }\end{array}$ \\
\hline Flammula peregrina (Fr.) Sacc. & BRA & Pegler (1997) \\
\hline Geophila hyperella (Fr.) Kühner \& Romagn. & ARG & $\begin{array}{l}\text { Wright \& Albertó (2002) as Psilocybe } \\
\text { hyperella }\end{array}$ \\
\hline Hypholoma aurantiacum (Cooke) Faus & ARG, BRA, CHI & $\begin{array}{l}\text { Silva et al. (2006, 2008); Rick (1961) as } \\
\text { Stropharia thrausta; Moreno \& Albertó } \\
\text { (1996), Meijer (2001, 2006), Sobestiansky } \\
\text { (2005) and George-Nascimento (2007) as S. } \\
\text { aurantiaca }\end{array}$ \\
\hline Hypholoma capnoides (Fr.) P. Kumm. & ARG, CHI, VEN & $\begin{array}{l}\text { Dennis (1961) and Niveiro \& Albertó (2012); } \\
\text { Singer (1969) as Naematoloma capnoides }\end{array}$ \\
\hline Hypholoma castilloi (Singer) Raithelh. & ARG & $\begin{array}{l}\text { Niveiro \& Albertó (2012); Singer (1969) as } \\
\text { Naematoloma castilloi }\end{array}$ \\
\hline Hypholoma confusum Dennis & VEN & Dennis (1961) \\
\hline Hypholoma elongatum (Pers.) Ricken & $\mathrm{ARG}, \mathrm{CHI}$ & $\begin{array}{l}\text { Singer (1969) as Naematoloma elongatipes; } \\
\text { Horak (1980) and Niveiro \& Albertó (2012) as } \\
\text { H. elongatipes. }\end{array}$ \\
\hline Hypholoma ericaeum (Pers.) Kühner & BRA, VEN & $\begin{array}{l}\text { Meijer (2001, 2006) and Silva et al. (2006, } \\
2008,2012) \text {; Singer (1953) and Pereira \& } \\
\text { Putzke (1990) as Naematoloma } \\
\text { subumbonatescens; Delgado \& Urdaneta } \\
\text { (2002) as N. ericaeum; Rick (1961) as } \\
\text { Stropharia ericaea. }\end{array}$ \\
\hline Hypholoma fasciculare (Huds.) P. Kumm. & $\mathrm{ARG}, \mathrm{BRA}$ & $\begin{array}{l}\text { Wright \& Albertó (2002) and Niveiro \& } \\
\text { Albertó (2012); Rick (1919, 1939, 1961) and } \\
\text { Singer (1969) as Naematoloma fasciculare }\end{array}$ \\
\hline Hypholoma fasciculare var. mitis Raithelh. & ARG & Niveiro \& Albertó (2012) \\
\hline Hypholoma frowardii (Speg.) Garrido & $\mathrm{ARG}, \mathrm{CHI}$ & $\begin{array}{l}\text { Niveiro \& Albertó (2012); Singer (1969) as } \\
\text { Pholiota frowardii }\end{array}$ \\
\hline Hypholoma intonsum Pass. & ARG & $\begin{array}{l}\text { Spegazzinni (1899) and Niveiro \& Albertó } \\
(2012)\end{array}$ \\
\hline Hypholoma lateritium (Schaeff.) P. Kumm. & $\mathrm{ARG}, \mathrm{CHI}, \mathrm{COL}$ & $\begin{array}{l}\text { Singer (1969) as Naematoloma sublateritium; } \\
\text { Wright \& Albertó (2002), Franco-Molano et } \\
\text { al. (2010) and Niveiro \& Albertó (2012) as } H \text {. } \\
\text { sublateritium }\end{array}$ \\
\hline Hypholoma megapotamicum (Speg.) Speg. & ARG & Spegazzinni (1899) and Niveiro \& Albertó \\
\hline
\end{tabular}




Taxa
Hypholoma nodipes Speg.
Hypholoma polylepidis Dennis
Hypholoma puiggarii (Speg.) Raithelh.
Hypholoma radicosoides Raithelh.
Hypholoma radicosum J.E. Lange
Hypholoma stellula Speg.
Hypholoma subviride (Berk. \& M.A. Curtis)
Dennis

Distribution

ARG

VEN

ARG, BRA, URU

ARG

CHI(?)

ARG

ARG, BRA, COL, CRC, CUB, DMA, GLP, JAM, MTQ, TTO, VEN

Hypholoma trinitensis (Dennis) Pegler

Hypholoma udum (Pers.) Quél.

Kuehneromyces cystidiosus Singer

Kuehneromyces macrosporus Singer

Kuehneromyces mutabilis (Schaeff.) Singer \& A.H. Sm.

Kuehneromyces nudus Singer

Kuehneromyces pseudoblattaria E. Horak

Kuehneromyces vinicolor (Pat.) Pegler

Leratiomyces ceres (Cooke \& Massee)

Spooner \& Bridge

Leratiomyces squamosus (Pers.) Bridge \& Spooner

Melanotus alpiniae (Berk.) Pilát

Melanotus brevisporus Singer

Melanotus cassiicolor (Berk.) Singer

Melanotus decapitatus Singer

Melanotus dumontii Singer

Melanotus eccentricus (Murrill) Singer

Melanotus gayi (Pilát) Singer

Melanotus heteroloma Singer

Melanotus fumosifolius (Murrill) Murrill

Melanotus matrisdei Singer

Melanotus musaecola (Berk. \& M.A. Curtis)

Singer

Melanotus patagonicus Singer

Melanotus polylepidis Singer

Melanotus subcuneifolius (Murrill) Singer

Melanotus subvariabilis (Speg.) Singer

Naematoloma amazonicum Singer

Naematoloma nudum Singer

Naucoria devia (Singer) Raithelh.

COL

ARG, CHI

ARG, CHI

BRA, JAM

ARG

ARG

GLP, MTQ

BRA

CRC

BRA(?) VEN(?)

BRA(?), VEN

BRA, COL

BOL

JAM

BOL

BRA, CUB

ARG

ARG

BRA, GRN, VEN

BRA

BRA ARG

\section{Reference}

(2012)

Niveiro \& Albertó (2012)

Dennis (1961)

Wright \& Albertó (2002), Meijer (2006) and Niveiro \& Albertó (2012); Spegazzini (1899) as Flammula puiggarii; Singer (1969) as Naematoloma puiggarii

Niveiro \& Albertó (2012)

Singer (1969) as Naematoloma radicosum

Spegazzinni (1899) and Niveiro \& Albertó (2012)

Dennis (1961), Pegler (1983, 1997), Bononi et al. (1984), Sotão et al. (1991), Ovrebo (1996), Cortez \& Coelho (2004) and Meijer (2006); Pegler (1987a) as Agaricus subviridis; FrancoMolano \& Uribe-Calle (2000) and Wright \& Albertó (2002) as Naematoloma subviride

ARG, BRA, MTQ, Pegler (1983, 1997), Lechner et al. (2006), TTO Bononi et al. (2008), Wright et al. (2008) and Niveiro \& Albertó (2012)

Franco-Molano \& Uribe-Calle (2000) as Naematoloma udum

Singer (1969), Horak (1980) and Niveiro \& Albertó (2012)

Singer (1969) and Niveiro \& Albertó (2012)

Rick (1938, 1961) and Dennis (1968)

Singer (1950) and Niveiro \& Albertó (2012)

Horak (1967) and Niveiro \& Albertó (2012)

Pegler (1983)

Silva et al. (2012); Cortez \& Silveira (2007b) and Silva et al. (2006) as Hypholoma aurantiacum

Halling \& Mueller (2005) as Psilocybe squamosa

BRA, COL, CUB, Dennis (1961), Pegler (1983, 1988, 1997), GLP, MTQ, TTO Capelari \& Maziero (1988), Franco-Molano \& Uribe-Calle (2000) and Meijer $(2001,2006)$ Meijer (2006)

ARG, CHI, COL, Singer (1969), Franco-Molano et al. (2010) and and Niveiro \& Albertó (2012) as $M$. cassiaecolor

Singer (1989) and Meijer (2001, 2006)

Singer (1989), Franco-Molano \& Uribe-Calle (2000) and Meijer (2001, 2006)

ARG, BRA(?), Pegler (1983), Meijer (2006) and Niveiro \& GLP, MTQ Albertó (2012)

ARG, CHI Singer (1969) and Niveiro \& Albertó (2012)

Singer (1989)

Dennis (1968)

Singer (1989)

Singer (1965) and Pegler (1987a)

Singer (1969) and Niveiro \& Albertó (2012)

Singer (1973) and Niveiro \& Albertó (2012)

Pegler (1983); Rick (1938, 1961) as Crepidotus scymnodes

ARG, BRA Singer (1950) and Niveiro \& Albertó (2012); Spegazzini (1899) as Claudopus subvariabilis
Singer (1973)

Singer (1973)

Wright \& Albertó (2002) 


\begin{tabular}{|c|c|c|}
\hline Taxa & Distribution & Reference \\
\hline Naucoria rigidipes Speg. & BRA & Pegler (1997) \\
\hline Pachylepyrium fulvidula (Singer) Singer & ARG & $\begin{array}{l}\text { Spegazzini (1925) and Niveiro \& Albertó } \\
(2012)\end{array}$ \\
\hline $\begin{array}{l}\text { Phaeogalera dissimulans (Berk. \& Broome) } \\
\text { Holec }\end{array}$ & BRA & Pegler (1997) as Flammula sordida \\
\hline Pholiota aberrans A.H. Sm. \& Hesler & COL, VEN & $\begin{array}{l}\text { Franco-Molano et al. (2010); Dennis (1961) as } \\
\text { Hypholoma anomalum }\end{array}$ \\
\hline Pholiota abstrusa (Fr.) Singer & ARG & Niveiro \& Albertó (2012) \\
\hline Pholiota agrocybiformis Singer & $\mathrm{CHI}$ & Singer (1969) \\
\hline Pholiota alnea Singer & ARG & $\begin{array}{l}\text { Raithelhuber }(1988,1991,2004) \text { and Niveiro } \\
\text { \& Albertó (2012) }\end{array}$ \\
\hline Pholiota apiahyna Speg. & $\mathrm{ARG}, \mathrm{BRA}, \mathrm{COL}$ & $\begin{array}{l}\text { Singer (1950, 1953), Raithelhuber (1988, } \\
\text { 1991, 2004), Pegler (1997), Franco-Molano et } \\
\text { al. (2010) and Niveiro \& Albertó (2012) }\end{array}$ \\
\hline Pholiota ascophora (Peck) Singer & ARG, CHI & $\begin{array}{l}\text { Singer (1969), Raithelhuber (1988, 1991, } \\
\text { 2004) and Niveiro \& Albertó (2012) }\end{array}$ \\
\hline Pholiota aurantioalbida Singer & ARG, BRA(?) & $\begin{array}{l}\text { Singer (1969), Horak (1980), Raithelhuber } \\
\text { (1988, 1991, 2004), Meijer (2001, 2006) and } \\
\text { Niveiro \& Albertó (2012) }\end{array}$ \\
\hline Pholiota aurivella (Batsch) P. Kumm. & CHI, VEN & Dennis (1961) and Singer (1969) \\
\hline Pholiota aurivella var. squarrosipes Singer & ARG & $\begin{array}{l}\text { Raithelhuber (1988, 1991, 2004) and Niveiro } \\
\text { \& Albertó (2012) }\end{array}$ \\
\hline Pholiota baeosperma Singer & $\mathrm{ARG}, \mathrm{CHI}$ & $\begin{array}{l}\text { Singer (1969), Raithelhuber (1988, 1991, } \\
\text { 2004), Gamundi \& Horak (2002) and Niveiro } \\
\text { \& Albertó (2012) }\end{array}$ \\
\hline Pholiota bicolor (Speg.) Singer & ARG, BRA & $\begin{array}{l}\text { Pegler (1997), Raithelhuber (1988, 1991, } \\
\text { 2004), Meijer (2001, 2006) and Niveiro \& } \\
\text { Albertó (2012) }\end{array}$ \\
\hline Pholiota calendulina Singer & ARG & $\begin{array}{l}\text { Raithelhuber (1988, 1991, 2004) and Niveiro } \\
\text { \& Albertó (2012) }\end{array}$ \\
\hline Pholiota chacoensis Speg. & ARG & $\begin{array}{l}\text { Spegazzini (1926b), Singer (1950) and } \\
\text { Niveiro \& Albertó (2012) }\end{array}$ \\
\hline Pholiota conissans (Fr.) Kuyp. \& Tjall. & BRA & Albuquerque et al. (2007) \\
\hline Pholiota discolor (Peck) Sacc. & DOM, GLP, TTO & Pegler (1983) \\
\hline Pholiota flavida (Schaeff.) Singer & ARG & $\begin{array}{l}\text { Spegazzini (1926b) and Niveiro \& Albertó } \\
\text { (2012) }\end{array}$ \\
\hline Pholiota formosa Speg. & ARG & $\begin{array}{l}\text { Spegazzini (1926b), Singer (1950) and } \\
\text { Niveiro \& Albertó (2012) }\end{array}$ \\
\hline Pholiota freindlingiae (Singer) Singer & $\mathrm{CHI}$ & Singer (1969) \\
\hline Pholiota glutinigera Singer & ARG & Singer (1959) and Niveiro \& Albertó (2012) \\
\hline Pholiota glutinosipes Singer & VEN & Dennis (1961) \\
\hline Pholiota gummosa (Lasch) Singer & BRA, JAM & Dennis (1968) and Pegler (1997) \\
\hline Pholiota gymnopiloides Raithelh. & ARG & $\begin{array}{l}\text { Raithelhuber (1974, 1988, 1991, 2004) and } \\
\text { Niveiro \& Albertó (2012) }\end{array}$ \\
\hline $\begin{array}{l}\text { Pholiota highlandensis (Peck) Quadr. \& } \\
\text { Lunghini }\end{array}$ & ARG, BRA, CHI & $\begin{array}{l}\text { Raithelhuber }(1974,1977,1988,1991,2004) \text {, } \\
\text { Meijer (2001, 2006), Wright \& Albertó (2002) } \\
\text { and Niveiro \& Albertó (2012); Singer (1969) } \\
\text { as P. carbonaria }\end{array}$ \\
\hline Pholiota irazuensis Singer & $\mathrm{CRC}$ & Singer (1989) \\
\hline Pholiota limonella (Peck) Sacc. & BRA & $\begin{array}{l}\text { Meijer }(2001,2006), \text { Sobestiansky (2005) and } \\
\text { Silva et al. (2012) }\end{array}$ \\
\hline Pholiota majalis Singer & $\mathrm{CHI}$ & Singer (1969) \\
\hline Pholiota marthae Singer & $\mathrm{CHI}$ & Singer (1969) \\
\hline Pholiota martinicensis Pat. & MTQ & Pegler (1983) \\
\hline Pholiota megalosperma Singer & ARG & $\begin{array}{l}\text { Singer (1969), Horak (1980), Raithelhuber } \\
(1988,1991,2004) \text { and Niveiro \& Albertó } \\
(2012)\end{array}$ \\
\hline Pholiota microcarpa Singer & ARG, CHI & $\begin{array}{l}\text { Singer (1969), Raithelhuber }(1977,1988 \text {, } \\
\text { 1991, 2004) and Niveiro \& Albertó }(2012)\end{array}$ \\
\hline Pholiota montana Singer & $\mathrm{ARG}, \mathrm{CHI}$ & $\begin{array}{l}\text { Singer (1969), Horak (1980), Raithelhuber } \\
(1988,1991,2004) \text { and Niveiro \& Albertó } \\
(2012)\end{array}$ \\
\hline
\end{tabular}




\begin{tabular}{|c|c|c|}
\hline Taxa & Distribution & Reference \\
\hline Pholiota myxacioides Singer & $\mathrm{CHI}$ & Singer (1969) \\
\hline Pholiota naucorioides Singer & BRA, COL & $\begin{array}{l}\text { Singer (1955), Meijer (2006) and Franco- } \\
\text { Molano et al. (2010) }\end{array}$ \\
\hline Pholiota novembris Singer & ARG & $\begin{array}{l}\text { Singer (1969), Raithelhuber (1977) and } \\
\text { Niveiro \& Albertó (2012) }\end{array}$ \\
\hline $\begin{array}{l}\text { Pholiota nubicola (Singer) Matheny \& P.-A. } \\
\text { Moreau }\end{array}$ & VEN & Dennis (1961) as Pachylepyrium nubicola \\
\hline Pholiota oblita Niveiro, Popoff \& Albertó & ARG & Niveiro et al. (2014) \\
\hline Pholiota ochrospora Raithelh. & ARG & $\begin{array}{l}\text { Raithelhuber (1974, 1988, 1991, 2004), } \\
\text { Wright \& Albertó (2002) and Niveiro \& } \\
\text { Albertó (2012) }\end{array}$ \\
\hline Pholiota polychroa (Berk.) Smith \& Brodie & BRA, MTQ & $\begin{array}{l}\text { Singer (1953), Pegler (1983) and Bononi et al. } \\
(2008)\end{array}$ \\
\hline Pholiota privigna (Speg.) Singer & $\begin{array}{l}\text { ARG, CHI, COL, } \\
\text { VEN }\end{array}$ & $\begin{array}{l}\text { Dennis (1961), Singer (1969), Horak (1980), } \\
\text { Raithelhuber (1988, 1991, 2004), Franco- } \\
\text { Molano \& Uribe-Calle (2000) and Niveiro \& } \\
\text { Albertó (2012) }\end{array}$ \\
\hline Pholiota psathyrelloides Singer & ARG & $\begin{array}{l}\text { Singer (1969), Horak (1980), Raithelhuber } \\
\text { (1977, 1988, 1991, 2004) and Niveiro \& } \\
\text { Albertó (2012) }\end{array}$ \\
\hline Pholiota pseudofascicularis Speg. & ARG & $\begin{array}{l}\text { Speggazini (1899, 1926b), Singer (1950), } \\
\text { Raithelhuber (1991, 2004), Wright \& Albertó } \\
\text { (2002) and Niveiro \& Albertó (2012) }\end{array}$ \\
\hline Pholiota rosea Rick & BRA & Rick $(1919,1928,1938,1961)$ \\
\hline Pholiota rudis Rick & BRA & Rick (1961) \\
\hline Pholiota spumosa (Fr.) Singer & ARG, BRA, CHI & $\begin{array}{l}\text { Speggazini (1926b), Singer (1969), Pegler } \\
\text { (1997), Wright \& Albertó (2002), Cortez \& } \\
\text { Coelho (2003), Silva et al. (2012) and Niveiro } \\
\text { \& Albertó (2012) }\end{array}$ \\
\hline Pholiota spumosa var. crassitunicata Singer & ARG & $\begin{array}{l}\text { Singer (1969), Raithelhuber (1974, 1977, } \\
\text { 1988, 1991, 2004) and Niveiro \& Albertó } \\
(2012)\end{array}$ \\
\hline Pholiota squarrosoides (Peck) Sacc. & BRA(?) & Meijer (2006) \\
\hline Pholiota subflammans (Speg.) Sacc. & ARG, CHI, URU & $\begin{array}{l}\text { Speggazini (1887a, 1887b), Singer (1950, } \\
\text { 1969), Raithelhuber (1977, 1988, 1991, 2004), } \\
\text { Horak (1980), Wright \& Albertó (2002) and } \\
\text { Niveiro \& Albertó (2012) }\end{array}$ \\
\hline Pholiota subsquarrosa (Fr.) Sacc. & BRA & Rick $(1938,1961)$ \\
\hline Pholiota tuberculosa (Schaeff.) P. Kumm. & BRA & Rick $(1907,1938,1961)$ \\
\hline Pholiota varzeae Singer & BRA & Singer (1989) \\
\hline $\begin{array}{l}\text { Protostropharia semiglobata } \quad \text { (Batsch) } \\
\text { Redhead, Moncalvo \& Vilgalys }\end{array}$ & $\begin{array}{l}\text { ARG, BRA, COL, } \\
\text { VEN }\end{array}$ & $\begin{array}{l}\text { Rick (1907, 1939, 1961), Spegazzini (1887a, } \\
\text { 1887b, 1899, 1926a, 1926b), Dennis (1961), } \\
\text { Singer (1969), Horak (1980), Raithelhuber } \\
\text { (1991, 2004), Stijve \& de Meijer (1993), } \\
\text { Guzmán et al. (1994), Pegler (1997), Franco- } \\
\text { Molano \& Uribe-Calle (2000), Meijer (2001, } \\
\text { 2006), Wright \& Albertó (2002), Cortez \& } \\
\text { Coelho (2004), Cortez \& Silveira (2008) and } \\
\text { Niveiro \& Albertó (2012) as Stropharia } \\
\text { semiglobata }\end{array}$ \\
\hline Psilocybe acutipilea (Speg.) Guzmán & BRA & $\begin{array}{l}\text { Guzmán (1978, 1995), Pegler (1997) and } \\
\text { Guzmán et al. (1998); Spegazzini (1899) as } \\
\text { Deconica acutipilea }\end{array}$ \\
\hline Psilocybe aequatoriae Singer & EQU & Singer (1978) and Guzmán (2004) \\
\hline Psilocybe aggericola Singer \& A.H. Sm. & ARG & Guzmán (1978) and Niveiro \& Albertó (2012) \\
\hline Psilocybe albofimbriata (Rick) Singer & BRA & Singer (1986) and Guzmán (1995) \\
\hline Psilocybe alnetorum (Singer) Singer & ARG, BRA & $\begin{array}{l}\text { Guzmán (1978, 1995), Raithelhuber (1988, } \\
\text { 1991, 2004), Stijve \& Meijer (1993), Meijer } \\
\text { (2006) and Niveiro \& Albertó (2012) }\end{array}$ \\
\hline Psilocybe andina Guzmán & $\mathrm{ECU}$ & Guzmán (1978) \\
\hline Psilocybe angulata (Berk. \& Broome) Singer & CHI, VEN & Singer (1969) and Guzmán (1978) \\
\hline Psilocybe angustipleurocystidiata Guzmán & $\mathrm{COL}$ & Guzmán (1983) and Guzmán et al. (1998) \\
\hline
\end{tabular}




\begin{tabular}{|c|c|c|}
\hline Taxa & Distribution & Reference \\
\hline $\begin{array}{l}\text { Psilocybe antioquiensis Guzmán, Saldarr., } \\
\text { Pineda, G. García \& L.-F. Velázquez }\end{array}$ & $\mathrm{COL}$ & $\begin{array}{l}\text { Guzmán et al. (1994, 1998), Guzmán (1995) } \\
\text { and Franco-Molano et al. (2010) }\end{array}$ \\
\hline Psilocybe araucana Singer & ARG & $\begin{array}{l}\text { Singer (1969), Raithelhuber (1977, 1988, } \\
\text { 1991, 2004), Guzmán (1978) and Niveiro \& } \\
\text { Albertó (2012) }\end{array}$ \\
\hline Psilocybe aztecorum var. aztecorum R. Heim & CRC & Sáenz et al. (1983) and Guzmán (1995) \\
\hline Psilocybe bispora Guzmán & COL & $\begin{array}{l}\text { Guzmán et al. (2007) and Franco-Molano et } \\
\text { al. (2010) }\end{array}$ \\
\hline Psilocybe blattariopsis (Speg.) Singer & BRA & $\begin{array}{l}\text { Guzmán (1978), Pegler (1997), Guzmán et al. } \\
\text { (1998) and Meijer (2006); Spegazzini (1899) } \\
\text { as Pholiotella blattariopsis }\end{array}$ \\
\hline Psilocybe brasiliensis Guzmán & BRA & $\begin{array}{l}\text { Guzmán (1978), Bononi et al. (1984), Pegler } \\
\text { (1997) and Guzmán et al. (1998) }\end{array}$ \\
\hline Psilocybe bullacea (Bull.) P. Kumm. & $\mathrm{COL}$ & $\begin{array}{l}\text { Guzmán et al. (1994) and Franco-Molano \& } \\
\text { Uribe-Calle (2000) }\end{array}$ \\
\hline $\begin{array}{l}\text { Psilocybe cabiensis Guzmán, M. Torres \& } \\
\text { Ram.-Guill. }\end{array}$ & $\mathrm{COL}$ & Guzmán et al. (2004a) \\
\hline $\begin{array}{l}\text { Psilocybe caeruleoannulata Singer ex } \\
\text { Guzmán }\end{array}$ & BRA, URU & $\begin{array}{l}\text { Guzmán (1978, 1983, 1995), Stijve \& Meijer } \\
\text { (1993), Pegler (1997), Guzmán et al. (1998), } \\
\text { Cortez \& Coelho (2004), Guzmán \& Cortez } \\
\text { (2004), Meijer (2006) and Silva et al. (2006, } \\
\text { 2008, 2012) }\end{array}$ \\
\hline Psilocybe caerulescens Murrill & $\begin{array}{l}\text { ARG, BRA, COL, } \\
\text { GUA, PAN, VEN }\end{array}$ & $\begin{array}{l}\text { Guzmán (1983, 1995), Stijve \& Meijer (1993), } \\
\text { Guzmán et al. (1998), Meijer (2006), López- } \\
\text { Quintero et al. (2011) and Niveiro \& Alberto } \\
\text { (2012) }\end{array}$ \\
\hline Psilocybe caesioannulata Singer & $\mathrm{CHI}$ & Singer (1969) and Guzmán (1978) \\
\hline Psilocybe callosa (Fr.) Quél. & $\mathrm{CHI}$ & Singer (1969) and Guzmán (1978) \\
\hline Psilocybe carbonaria Singer & $\mathrm{CHI}$ & $\begin{array}{l}\text { Guzmán }(1978,1983) \text { and Guzmán et al. } \\
\text { (1998) }\end{array}$ \\
\hline $\begin{array}{l}\text { Psilocybe caribaea Guzmán, T. J. Baroni \& } \\
\text { Tapia }\end{array}$ & MTQ, PRI & $\begin{array}{l}\text { Guzmán et al. (2003); Pegler (1983) as } P \text {. } \\
\text { caerulescens }\end{array}$ \\
\hline Psilocybe chilensis Singer & $\mathrm{BRA}(?), \mathrm{CHI}$ & $\begin{array}{l}\text { Singer (1969), Guzmán (1978), Stijve \& } \\
\text { Meijer (1993) and Meijer (2006) }\end{array}$ \\
\hline Psilocybe chrysocystidiata Singer & $\mathrm{ARG}, \mathrm{BOL}$ & $\begin{array}{l}\text { Singer (1973), Guzmán (1978), Raithelhuber } \\
(1991,2004) \text { and Niveiro \& Alberto (2012) }\end{array}$ \\
\hline Psilocybe clavata Guzmán & $\mathrm{COL}$ & Guzmán (1983) \\
\hline Psilocybe collybioides Singer \& A.H. Sm. & ARG & $\begin{array}{l}\text { Guzmán }(1978,1983) \text { and Niveiro \& Alberto } \\
\text { (2012) }\end{array}$ \\
\hline Psilocybe columbiana Guzmán & COL & $\begin{array}{l}\text { Guzmán (1978, 1983), Guzmán et al. (1994, } \\
\text { 1998) and Franco-Molano \& Uribe-Calle } \\
(2000)\end{array}$ \\
\hline Psilocybe cordispora $\mathrm{R}$. Heim & $\mathrm{BIZ}$ & Guzmán et al. (1998) \\
\hline Psilocybe cordobensis Singer & ARG & $\begin{array}{l}\text { Singer (1973), Guzmán (1978), Raithelhuber } \\
\text { (1988, 1991, 2004) and Niveiro \& Albertó } \\
\text { (2012) }\end{array}$ \\
\hline Psilocybe crobula var. crobula (Fr.) Singer & BRA(?) & Stijve \& Meijer (1993) and Meijer (2006) \\
\hline Psilocybe cubensis (Earle) Singer & $\begin{array}{l}\text { ARG, BIZ, BRA, } \\
\text { COL, CRC, CUB, } \\
\text { DOM, DMA, ECU, } \\
\text { ESA, GLP, GUA, } \\
\text { GUF, MTQ, PAN, } \\
\text { PER, PRI, TTO, } \\
\text { VEN }\end{array}$ & $\begin{array}{l}\text { Singer (1953), Guzmán (1978, 1983, 1995), } \\
\text { Reid et al. (1980), Pegler (1983), Sáenz et al. } \\
\text { (1983), Rodriguez-Gallart (1989), Navarro \& } \\
\text { Betancourt (1992), Stijve \& Meijer (1993), } \\
\text { Courtecuisse et al. (1996), Franco-Molano \& } \\
\text { Uribe-Calle (2000), Guzmán et al. (1998), } \\
\text { Delgado \& Urdaneta (2002), Wright \& } \\
\text { Albertó (2002), Cortez \& Coelho (2004), } \\
\text { Meijer (2006), Silva et al. (2006, 2008), } \\
\text { Wartchow et al. (2007), Laessoe \& Petersen } \\
\text { (2008), Piepenbring (2008), Flores Arzú et al. } \\
\text { (2012) and Niveiro \& Albertó (2012); } \\
\text { Montagne (1856) as Agaricus rhytopilus; Rick } \\
\text { (1930, 1939, 1961) as Stropharia } \\
\text { subcyanescens; Pegler (1987b) as S. cubensis }\end{array}$ \\
\hline
\end{tabular}




\begin{tabular}{|c|c|c|}
\hline Taxa & Distribution & Reference \\
\hline Psilocybe cyanescens Wakef. & GUA & Flores Arzú et al. (2012) \\
\hline Psilocybe dumontii Singer ex Guzmán & PAN & Guzmán (1978) and Guzmán et al. (1998) \\
\hline Psilocybe dunicola (Speg.) Singer & ARG & $\begin{array}{l}\text { Spegazzini (1899), Singer (1969), Guzmán } \\
\text { (1978), Wright \& Albertó (2002) and Niveiro } \\
\text { \& Albertó (2012) }\end{array}$ \\
\hline Psilocybe egonii Guzmán \& T. J. Baroni & PRI & Guzmán et al. (2003) \\
\hline Psilocybe farinacea Rick ex Guzmán & BRA & $\begin{array}{l}\text { Guzmán (1978) and Guzmán et al. (1998); } \\
\text { Rick (1961) as Naucoria albofimbriata and } N \text {. } \\
\text { tenax }\end{array}$ \\
\hline Psilocybe februaria Singer & BOL & Singer (1989) \\
\hline Psilocybe fimetaria (P.D. Orton) Watling & $\mathrm{CHI}$ & $\begin{array}{l}\text { Singer (1969), Guzmán (1983) and Guzmán et } \\
\text { al. (1998) }\end{array}$ \\
\hline Psilocybe fimicola Guzmán & $\mathrm{COL}$ & $\begin{array}{l}\text { Guzmán et al. (1994) and Franco-Molano \& } \\
\text { Uribe-Calle (2000) }\end{array}$ \\
\hline Psilocybe flammuliformis Singer & $\mathrm{CHI}$ & Singer (1969) and Guzmán (1978) \\
\hline Psilocybe fuegiana (E. Horak) Singer & ARG, PER & $\begin{array}{l}\text { Spegazzini (1926b), Singer (1969), Guzmán } \\
\text { (1978), Horak (1980), Yokoyama (1987), } \\
\text { Raithelhuber (1988, 1991, 2004) and Niveiro } \\
\text { \& Albertó (2012) }\end{array}$ \\
\hline Psilocybe fuliginosa (Murrill) A.H. Sm. & JAM & Guzmán (1983) and Guzmán et al. (1998) \\
\hline Psilocybe furtadoana Guzmán & BRA & $\begin{array}{l}\text { Guzmán (1978), Bononi et al. (1984), Pegler } \\
\text { (1997) and Guzmán et al. (1998) }\end{array}$ \\
\hline Pholiota galapagensis Pegler & $\mathrm{ECU}$ & Reid et al. (1980) \\
\hline $\begin{array}{l}\text { Psilocybe guatapensis Guzmán, Saldarr., } \\
\text { Pineda, G. García \& L.-F. Velázquez }\end{array}$ & $\mathrm{COL}$ & $\begin{array}{l}\text { Guzmán et al. (1994, 1998) and Franco- } \\
\text { Molano et al. (2010) }\end{array}$ \\
\hline $\begin{array}{l}\text { Psilocybe guilartensis Guzmán, F. Tapia \& } \\
\text { Nieves-Riv. }\end{array}$ & DOM, PRI & Guzmán et al. (1997, 1998, 2003) \\
\hline $\begin{array}{l}\text { Psilocybe heliconiae Guzmán, Saldarr., } \\
\text { Pineda, G. García \& L.-F. Velázquez }\end{array}$ & $\mathrm{COL}$ & $\begin{array}{l}\text { Guzmán et al. (1994, 1998) and Franco- } \\
\text { Molano et al. (2010) }\end{array}$ \\
\hline Psilocybe hoogshagenii R. Heim & ARG, BRA, COL & $\begin{array}{l}\text { Guzmán (1978), Stijve \& Meijer (1993), } \\
\text { Guzmán et al. (1998), Meijer (2006) and } \\
\text { Niveiro \& Albertó (2012) }\end{array}$ \\
\hline Psilocybe horakii Guzmán & ARG & Guzmán (1978) and Niveiro \& Albertó (2012) \\
\hline Psilocybe inquilina (Fr.: Fr.) Bres. & $\begin{array}{l}\text { ARG, BRA, CHI, } \\
\text { URU }\end{array}$ & $\begin{array}{l}\text { Spegazzini (1909), Guzmán (1978), } \\
\text { Raithelhuber (2004), Meijer (2006) and Silva } \\
\text { et al. (2008); Singer (1969), Raithelhuber } \\
\text { (1977, 1988, 1991), Wright \& Albertó (2002) } \\
\text { and Niveiro \& Albertó (2012) as P. ecbola; } \\
\text { Silva et al. (2006) as P. muscorum }\end{array}$ \\
\hline Psilocybe jujuyensis Singer & ARG & $\begin{array}{l}\text { Singer (1973), Guzmán (1978), Raithelhuber } \\
(1991,2004) \text { and Niveiro \& Albertó (2012) }\end{array}$ \\
\hline Psilocybe lazoi Singer & $\mathrm{CHI}$ & $\begin{array}{l}\text { Singer }(1969,1986) \text { and Guzmán et al. (1998); } \\
\text { Guzmán }(1978) \text { as P. zapotecorum }\end{array}$ \\
\hline $\begin{array}{l}\text { Psilocybe liniformans var. americana } \\
\text { Guzmán \& Stamets }\end{array}$ & $\mathrm{CHI}$ & Guzmán (1983) \\
\hline Psilocybe mammillata (Murrill) A.H. Sm. & BOL, JAM & $\begin{array}{l}\text { Dennis (1968), Guzmán (1983) and Guzmán } \\
\text { et al. (1998) }\end{array}$ \\
\hline Psilocybe marthae Singer & $\mathrm{CHI}$ & Singer (1969) and Guzmán (1978) \\
\hline Psilocybe meridensis Guzmán & VEN & Guzmán (1995) and Guzmán et al. (1998) \\
\hline Psilocybe mesospora Singer & ARG & $\begin{array}{l}\text { Singer (1969), Guzmán (1978), Raithelhuber } \\
(1988,1991) \text { and Niveiro \& Albertó (2012) }\end{array}$ \\
\hline Psilocybe mexicana $\mathrm{R}$. Heim & COL, CRC, GUA & $\begin{array}{l}\text { Flores Arzú et al.(2012); Sáenz et al. (1983) as } \\
P \text {. cf. mexicana; Guzmán et al. (1994, 1998) } \\
\text { and Franco-Molano et al. (2010) as P. } \\
\text { subacutipilea }\end{array}$ \\
\hline Psilocybe microcystidiata Guzmán \& Bononi & BRA & Guzmán et al. $(1984,1998)$ \\
\hline Psilocybe muliercula Singer \& A.H.Sm. & $\mathrm{BRA}(?)$ & Meijer (2006) \\
\hline Psilocybe omiumsanctorum Singer & $\mathrm{ARG}, \mathrm{CHI}$ & $\begin{array}{l}\text { Singer (1969), Raithelhuber (1977, 1988, } \\
\text { 1991), Guzmán (1978) and Niveiro \& Albertó } \\
\text { (2012) }\end{array}$ \\
\hline Psilocybe pallidispora (Murrill) A.H. Sm. & JAM & Dennis (1968) \\
\hline Psilocybe panaeoliformis Murrill & ECU & Guzmán (1978) \\
\hline
\end{tabular}




\begin{tabular}{|c|c|c|}
\hline Taxa & Distribution & Reference \\
\hline $\begin{array}{l}\text { Psilocybe paulensis (Guzmán \& Bononi) } \\
\text { Guzmán }\end{array}$ & BRA & $\begin{array}{l}\text { Guzmán et al. (1984, 1998) and Guzmán } \\
\text { (1995) }\end{array}$ \\
\hline Psilocybe paupera Singer & BRA & $\begin{array}{l}\text { (Singer 1953), Guzmán (1978), Stijve \& } \\
\text { Meijer (1993) and Guzmán et al. (1998) }\end{array}$ \\
\hline Psilocybe peladae Singer & $\mathrm{CHI}$ & Singer (1969) and Guzmán (1978) \\
\hline $\begin{array}{l}\text { Psilocybe pelliculosa (A.H. Sm.) Singer \& } \\
\text { A.H. Sm. }\end{array}$ & $\mathrm{CHI}$ & Guzmán (1978) \\
\hline Psilocybe pericystis Singer & BRA & Singer (1989) and Guzmán et al. (1998) \\
\hline Psilocybe peruviana Singer & COL, PER & Singer (1959) and Guzmán (1978) \\
\hline Psilocybe phyllogena (Sacc.) Peck & & \\
\hline Psilocybe pintonii Guzmán & $\mathrm{COL}$ & $\begin{array}{l}\text { Guzmán (1978, 1983), Guzmán et al. (1994) } \\
\text { and Franco-Molano \& Uribe-Calle (2000) }\end{array}$ \\
\hline Psilocybe pleurocystidiosa Guzmán & $\mathrm{COL}$ & Guzmán (1983) \\
\hline $\begin{array}{l}\text { Psilocybe plutonia (Berk. \& M.A. Curtis) } \\
\text { Sacc. }\end{array}$ & $\begin{array}{l}\text { BRA, COL, CUB, } \\
\text { GLP, MTQ, VEN }\end{array}$ & $\begin{array}{l}\text { Dennis }(1961,1970) \text {, Guzmán (1978, 1983), } \\
\text { Pegler (1983, 1987a) and Guzmán et al. } \\
(1998,2004 a)\end{array}$ \\
\hline $\begin{array}{l}\text { Psilocybe portoricensis Guzmán, Nieves-Riv. } \\
\text { \& F. Tapia }\end{array}$ & PRI & Guzmán et al. $(1997,1998)$ \\
\hline Psilocybe praetervisa Singer & ARG & $\begin{array}{l}\text { Singer (1969), Guzmán (1978), Raithelhuber } \\
\text { (1991, 2004) and Niveiro \& Albertó (2012) }\end{array}$ \\
\hline Psilocybe pseudobullacea (Petch) Pegler & VEN & $\begin{array}{l}\text { Marcano et al. (1994) and Guzmán et al. } \\
\text { (1998) }\end{array}$ \\
\hline Psilocybe pteridophytorum Singer & ARG & $\begin{array}{l}\text { Singer (1959), Guzmán (1978) and Niveiro \& } \\
\text { Albertó (2012) }\end{array}$ \\
\hline $\begin{array}{l}\text { Psilocybe ramulosa (Guzmán \& Bononi) } \\
\text { Guzmán }\end{array}$ & BRA & $\begin{array}{l}\text { Guzmán (1995), Stijve \& Meijer (1993) and } \\
\text { Guzmán et al. (1998); Guzmán et al. (1984), } \\
\text { Pegler (1997) and Bononi et al. (2008) as P. } \\
\text { zapotecorum var. ramulosus }\end{array}$ \\
\hline Psilocybe rickii Guzmán \& Cortez & BRA & Guzmán \& Cortez (2005) \\
\hline $\begin{array}{l}\text { Psilocybe scatigena (Berk. \& M.A. Curtis) } \\
\text { Guzmán }\end{array}$ & CUB & Guzmán (1983) and Pegler (1987a) \\
\hline Psilocybe sclerotifera (Speg.) Singer & ARG & Guzmán (1978) \\
\hline $\begin{array}{l}\text { Psilocybersemiangustipleurocystidiata } \\
\text { Guzmán, Ram.-Guill. \& M. Torres }\end{array}$ & $\mathrm{COL}$ & Guzmán et al. (2004a) \\
\hline Psilocybe semilanceata (Fr.) P. Kumm. & $\mathrm{CHI}$ & $\begin{array}{l}\text { Singer (1969), Guzmán (1978, 1983) and } \\
\text { Guzmán et al. (1998) }\end{array}$ \\
\hline Psilocybe septembris (Singer) Singer & $\mathrm{CHI}$ & $\begin{array}{l}\text { Guzmán (1978); Singer (1969) as Pholiota } \\
\text { septembris }\end{array}$ \\
\hline Psilocybe sierrae Singer & $\mathrm{CHI}$ & $\begin{array}{l}\text { Singer (1969, 1986), Guzmán (1978, 1983) } \\
\text { and Guzmán et al. (1998) }\end{array}$ \\
\hline Psilocybe singeriana Guzmán & BRA & Guzmán (1983) and Meijer (2006) \\
\hline Psilocybe squalens (Fr.) Sacc. & ARG & $\begin{array}{l}\text { Spegazzini (1926b) and Niveiro \& Albertó } \\
(2012)\end{array}$ \\
\hline Psilocybe squarrosipes Singer & ARG & $\begin{array}{l}\text { Singer (1959), Guzmán (1978) and Niveiro \& } \\
\text { Albertó (2012) }\end{array}$ \\
\hline Psilocybe strictipes Singer \& A.H. Sm. & $\mathrm{CHI}$ & $\begin{array}{l}\text { Singer (1969), Guzmán (1983) and Guzmán et } \\
\text { al. (1998) }\end{array}$ \\
\hline Psilocybe subaeruginascens Höhn. & BRA & Silva et al. (2014) \\
\hline Psilocybe subalnetorum Guzmán \& E. Horak & ARG & $\begin{array}{l}\text { Guzmán \& Horak (1978) and Niveiro \& } \\
\text { Albertó (2012) }\end{array}$ \\
\hline Psilocybe subcubensis Guzmán & $\begin{array}{l}\text { BOL, COL, ECU, } \\
\text { ESA, HON, PRI, } \\
\text { VEN }\end{array}$ & $\begin{array}{l}\text { Guzmán (1978, 1983), Velazquez et al. } \\
\text { (1991), Navarro \& Betancourt (1992), } \\
\text { Guzmán et al. (1994, 1997, 1998) and Franco- } \\
\text { Molano \& Uribe-Calle (2000) }\end{array}$ \\
\hline $\begin{array}{l}\text { Psilocybe subheliconiae Guzmán, Ram.-Guill. } \\
\& \text { M. Torres }\end{array}$ & $\mathrm{COL}$ & Guzmán et al. (2004a) \\
\hline $\begin{array}{l}\text { Psilocybe subhoogshagenii Guzmán, M. } \\
\text { Torres \& Ram.-Guill. }\end{array}$ & $\mathrm{COL}$ & Guzmán et al. (2004a) \\
\hline Psilocybe subhyperella Singer & $\mathrm{BRA}(?), \mathrm{COL}$ & $\begin{array}{l}\text { Singer (1973) and Guzmán (1978); Franco- } \\
\text { Molano \& Uribe-Calle (2000) and Meijer } \\
\text { (2006) as P. castanella var. subhyperella }\end{array}$ \\
\hline Psilocybe subpsilocybioides Guzmán, Lodge & PRI & Guzmán et al. (2003) \\
\hline
\end{tabular}




\begin{tabular}{|c|c|c|}
\hline Taxa & Distribution & Reference \\
\hline \multicolumn{3}{|l|}{ \& Cantrell } \\
\hline Psilocybe subtropicalis Guzmán & GUA & Guzmán (1995) and Flores Arzú et al. (2012) \\
\hline Psilocybe subyungensis Guzmán & BRA(?), VEN & $\begin{array}{l}\text { Guzmán (1978, 1983), Stijve \& Meijer (1993), } \\
\text { Guzmán et al. (1998) and Meijer (2006) }\end{array}$ \\
\hline Psilocybe tortipes Speg. & ARG, BRA & $\begin{array}{l}\text { Rick (1906, 1908, 1939, 1961), Theissen } \\
\text { (1912) and Guzmán (1978); Niveiro \& } \\
\text { Albertó (2002) as Naematoloma tortipes }\end{array}$ \\
\hline Psilocybe trufemiae Guzmán \& Bononi & BRA & $\begin{array}{l}\text { Bononi et al. (1984), Guzmán et al. (1984) and } \\
\text { Pegler (1997) }\end{array}$ \\
\hline Psilocybe uruguayensis Singer ex Guzmán & BRA, URU & $\begin{array}{l}\text { Guzmán (1978, 1983), Stijve \& Meijer (1993) } \\
\text { and Guzmán et al. (1998) }\end{array}$ \\
\hline Psilocybe valdiviensis Singer & BRA(?), CHI & $\begin{array}{l}\text { Singer (1969), Guzmán (1978), Stijve \& } \\
\text { Meijer (1993) and Meijer (2006) }\end{array}$ \\
\hline Psilocybe venezuelana Dennis & $\begin{array}{l}\text { BRA, GLP, MTQ, } \\
\text { VEN }\end{array}$ & $\begin{array}{l}\text { Dennis (1961), Guzmán (1978), Pegler (1997, } \\
\text { 1983), Stijve \& Meijer (1993), Meijer (2006) } \\
\text { and Bononi et al. (2008) }\end{array}$ \\
\hline Psilocybe wrightii Guzmán & ARG, BRA, COL & $\begin{array}{l}\text { Guzmán (1978, 1983), Guzmán \& Cortez } \\
\text { (2004), Silva et al. (2006, 2008, 2012), } \\
\text { Franco-Molano et al. (2010) and Niveiro \& } \\
\text { Albertó (2012) }\end{array}$ \\
\hline Psilocybe yungensis Singer \& A.H. Sm. & $\begin{array}{l}\text { BOL, COL, ECU, } \\
\text { PER(?) }\end{array}$ & $\begin{array}{l}\text { Guzmán (1978, 1983), Guzmán et al. (1994, } \\
\text { 1998) and Franco-Molano \& Uribe-Calle } \\
(2000)\end{array}$ \\
\hline $\begin{array}{l}\text { Psilocybe zapotecoantillarum Guzmán, T. J. } \\
\text { Baroni \& Lodge }\end{array}$ & PRI & Guzmán et al. (2003) \\
\hline $\begin{array}{l}\text { Psilocyberapotecocaribaea Guzmán, } \\
\text { Ramírez-Guillén \& T. J. Baroni }\end{array}$ & MTQ & $\begin{array}{l}\text { Guzmán et al. (2003); Pegler (1983) as } P \text {. } \\
\text { yungensis }\end{array}$ \\
\hline Psilocybe zapotecorum Heim. & $\begin{array}{l}\text { ARG, BRA, CHI, } \\
\text { COL, ECU, GUA, } \\
\text { PER, VEN }\end{array}$ & $\begin{array}{l}\text { Guzmán (1978, 1983, 2012), Stijve \& Meijer } \\
\text { (1993), Guzmán et al. (1994, 1998), Franco- } \\
\text { Molano \& Uribe-Calle (2000), Wright \& } \\
\text { Albertó (2002), Sobestiansky (2005), Meijer } \\
\text { (2006), Silva et al. (2012) and Flores Arzú et } \\
\text { al. (2012) }\end{array}$ \\
\hline $\begin{array}{l}\text { Psilocybe zoncuantlensis Guzmán \& Ram.- } \\
\text { Guillén }\end{array}$ & $\operatorname{BRA}(?)$ & Meijer (2006) \\
\hline $\begin{array}{l}\text { Stagnicola perplexa (P.D. Orton) Redhead \& } \\
\text { A.H. Sm. }\end{array}$ & ARG & Singer (1969) and Niveiro et al. (2012) \\
\hline $\begin{array}{l}\text { Stropharia acanthocystis Cortez \& R.M. } \\
\text { Silveira }\end{array}$ & BRA & $\begin{array}{l}\text { Cortez \& Silveira (2007a) and Silva et al. } \\
\text { (2012) }\end{array}$ \\
\hline Stropharia aeruginosa (Curtis) Quél. & BRA & Cortez \& Silveira (2008) \\
\hline $\begin{array}{l}\text { Stropharia aeruginosa var. neuquenensis } \\
\text { M.M. Moser \& Singer }\end{array}$ & ARG & $\begin{array}{l}\text { Singer (1969), Raithelhuber (1977, 1988, } \\
\text { 1991, 2004) and Niveiro \& Albertó (2012) }\end{array}$ \\
\hline $\begin{array}{l}\text { Stropharia agaricoides P.S. Silva, Cortez \& } \\
\text { R.M. Silveira }\end{array}$ & BRA & Silva et al. (2009) \\
\hline Stropharia agrocyboides (Raithelh.) Raithelh. & ARG & $\begin{array}{l}\text { Raithelhuber (1988, 1991, 2004) and Niveiro } \\
\text { \& Albertó (2012); Wright \& Albertó (2002) as } \\
\text { Hypholoma agrocyboides }\end{array}$ \\
\hline $\begin{array}{l}\text { Stropharia albivelata (Murrill) Norvell \& } \\
\text { Redhead }\end{array}$ & $\operatorname{BRA}(?)$ & Meijer (2006) \\
\hline $\begin{array}{l}\text { Stropharia alcis var. austrobrasiliensis Cortez } \\
\text { \& R.M. Silveira }\end{array}$ & BRA & $\begin{array}{l}\text { Cortez \& Silveira (2008); Silva et al. (2006, } \\
\text { 2008) as S. alcis }\end{array}$ \\
\hline $\begin{array}{l}\text { Stropharia apiahyna (Speg.) Cortez \& R.M. } \\
\text { Silveira }\end{array}$ & BRA, COL & $\begin{array}{l}\text { Singer (1978) and Cortez \& Silveira (2008); } \\
\text { Singer (1953) as Pholiota apiahyna; Pegler } \\
\text { (1997) as Naucoria apiahyensis }\end{array}$ \\
\hline Stropharia araucariae Cortez \& R.M. Silveira & BRA & $\begin{array}{l}\text { Cortez \& Silveira (2008) and Silva et al. } \\
\text { (2012) }\end{array}$ \\
\hline Stropharia bruchii Speg. & $\mathrm{ARG}$ & $\begin{array}{l}\text { Spegazzini (1926a) and Niveiro \& Albertó } \\
\text { (2012) }\end{array}$ \\
\hline Stropharia coronilla (Bull.) Quél. & ARG, BRA & $\begin{array}{l}\text { Rick (1907, 1939, 1961), Spegazzini (1926a), } \\
\text { Batista \& Bezerra (1960), Singer (1969), } \\
\text { Raithelhuber (1974, 1988, 1991, 2004), Stijve } \\
\text { \& de Meijer (1993), Wright \& Albertó (2002), }\end{array}$ \\
\hline
\end{tabular}




\begin{tabular}{|c|c|c|}
\hline Taxa & Distribution & Reference \\
\hline & & $\begin{array}{l}\text { Cortez \& Coelho (2004), Meijer (2006), Silva } \\
\text { et al. (2006, 2008) and Niveiro \& Albertó } \\
(2012)\end{array}$ \\
\hline Stropharia coronilla var. magnispora & ARG & $\begin{array}{l}\text { Raithelhuber }(2000,2004) \text { and Niveiro \& } \\
\text { Albertó (2012) }\end{array}$ \\
\hline Stropharia dorsipora Esteve-Rav. \& Barrasa & BRA & Cortez \& Silveira (2008) \\
\hline Stropharia earlei Norvell \& Redhead & $\begin{array}{l}\text { BRA, CRC, CUB, } \\
\text { GRN }\end{array}$ & $\begin{array}{l}\text { Cortez \& Silveira }(2008) \text { and Silva et al. } \\
\text { (2012); Pegler }(1983,1987 b, 1997) \text { as } \\
\text { Pholiota cubensis }\end{array}$ \\
\hline Stropharia flocculosipes Singer & $\mathrm{CHI}$ & Singer (1969) \\
\hline Stropharia lasseri Dennis & VEN & Dennis (1961) \\
\hline Stropharia luteonitens (Fr.) Quél. & ARG & $\begin{array}{l}\text { Spegazzini (1899) and Niveiro \& Albertó } \\
\text { (2012); Singer (1969) as S. umbonatescens }\end{array}$ \\
\hline $\begin{array}{l}\text { Stropharia melanosperma (Bull. ex Pers.: Fr.) } \\
\text { Gillet }\end{array}$ & ARG, BRA & $\begin{array}{l}\text { Horak (1967), Wright \& Albertó (2002), } \\
\text { Raithelhuber (2004), Cortez \& Coelho (2008) } \\
\text { and Niveiro \& Albertó (2012) }\end{array}$ \\
\hline Stropharia mitorhiza Speg. & ARG & $\begin{array}{l}\text { Spegazzini (1926b) and Niveiro \& Albertó } \\
\text { (2012) }\end{array}$ \\
\hline Stropharia pampeana Speg. & ARG & $\begin{array}{l}\text { Spegazzini (1899) and Niveiro \& Albertó } \\
(2012)\end{array}$ \\
\hline Stropharia pendolae (Speg.) Singer & ARG & $\begin{array}{l}\text { Spegazzini (1926b), Raithelhuber (1991) and } \\
\text { Niveiro \& Albertó (2012) }\end{array}$ \\
\hline Stropharia purpureoaurantiaca Raithelh. & ARG & $\begin{array}{l}\text { Raithelhuber (1974, 1988, 1991, 2004) and } \\
\text { Niveiro \& Albertó (2012) }\end{array}$ \\
\hline $\begin{array}{l}\text { Stropharia purpureoaurantiaca var. } \\
\text { magnispora Raithelh. }\end{array}$ & ARG & $\begin{array}{l}\text { Raithelhuber (1974, 1991, 2004) and Niveiro } \\
\text { \& Albertó (2012) }\end{array}$ \\
\hline Stropharia purpureoviolacea Raithelh. & ARG & $\begin{array}{l}\text { Raithelhuber (1974, 1988, 1991, 2004), } \\
\text { Wright \& Albertó (2002) and Niveiro \& } \\
\text { Albertó (2012) }\end{array}$ \\
\hline Stropharia rugosoannulata Farl. Ex Murrill & ARG, BRA & $\begin{array}{l}\text { Spegazzini (1889), Raithelhuber (1974, 1988, } \\
\text { 2004), Stijve \& de Meijer (1993), Pegler } \\
\text { (1997), Wright \& Albertó (2002), Cortez \& } \\
\text { Coelho (2004), Meijer (2006), Wright et al. } \\
\text { (2008), Silva et al. (2012) and Niveiro \& } \\
\text { Albertó (2012); Spegazzinni (1899) as } \\
\text { Flammula puiggarii; Rick (1961) as Anellaria } \\
\text { sanguineopurpurea }\end{array}$ \\
\hline $\begin{array}{l}\text { Stropharia rugosoannulata var. farlowiana } \\
\text { (Raithelh.) Raithelh. }\end{array}$ & ARG & $\begin{array}{l}\text { Raithelhuber (2004) and Niveiro \& Albertó } \\
\text { (2012) }\end{array}$ \\
\hline Stropharia stercoraria (Bull.: Fr.) Quél. & ARG, BRA & $\begin{array}{l}\text { Rick (1939, 1961) and Wright \& Albertó } \\
(2002)\end{array}$ \\
\hline Stropharia trinitensis (Dennis) Cortez & BRA & $\begin{array}{l}\text { Pegler (1997) as Hypholoma trinitense; Meijer } \\
\text { (2006) as Pholiota trinitense. }\end{array}$ \\
\hline $\begin{array}{l}\text { Stropharia umbonescens (Britzelm.) Sacc. \& } \\
\text { Traverso }\end{array}$ & ARG & $\begin{array}{l}\text { Singer (1969), Raithelhuber }(1991,2004) \text { and } \\
\text { Niveiro \& Albertó (2012) }\end{array}$ \\
\hline Stropharia varzeae (Singer) Cortez & BRA & Cortez (2008) \\
\hline $\begin{array}{l}\text { Stropharia venusta P.S. Silva, Cortez \& R.M. } \\
\text { Silveira }\end{array}$ & BRA & Silva et al. $(2009,2012)$ \\
\hline Stropharia viloriana (Dennis) Singer & VEN & Dennis (1961) as Hypholoma vilorianum \\
\hline \multicolumn{3}{|l|}{ Excluded species } \\
\hline Naucoria pusillima Speg. & BRA & Pegler (1997) \\
\hline Pholiota digiloi Singer & ARG & $\begin{array}{l}\text { Raithelhuber }(1988,1991,2004) \text { and Niveiro } \\
\text { \& Albertó (2012) }\end{array}$ \\
\hline Pholiota meliaphila Raithel. & ARG & $\begin{array}{l}\text { Raithelhuber }(2000,2004) \text { and Niveiro \& } \\
\text { Albertó (2012) }\end{array}$ \\
\hline Pholiota squarrosa Murr. & BRA & Rick $(1938,1961)$ \\
\hline Pholiota subfascicularis Speg. & BRA & Rick $(1907,1938,1961)$ \\
\hline Psilocybe heterosticha (Fr.) Singer & ARG, BRA & $\begin{array}{l}\text { Singer (1969), Guzmán (1978) and Pegler } \\
\text { (1997) }\end{array}$ \\
\hline Psilocybe uda (Pers.) Gillet & ARG & Horak (1967) and Niveiro \& Albertó (2012) \\
\hline Stropharia crassa Rick & BRA & Rick $(1907,1939,1961)$ \\
\hline
\end{tabular}




\begin{tabular}{lll}
\hline Taxa & Distribution & Reference \\
\hline Hypholoma intonsum Pass. & BRA & Cortez \& Silveira (2008) \\
Stropharia inuncta ss. Rick & BRA & Cortez \& Silveira (2008) \\
Stropharia mephistopheles Cooke & BRA & Rick (1907, Cortez \& Silveira (2008) \\
\hline
\end{tabular}

*Countries: $\mathrm{ARG}=$ Argentina; $\mathrm{BIZ}=$ Belize; $\mathrm{BRA}=$ Brazil; $\mathrm{BOL}=$ Bolivia CHI = Chile COL = Colombia CRC = Costa Rica; CUB = Cuba; DMA = Dominica; DOM = Dominican Republic; ECU = Ecuador; ESA = El Salvador; GLP = Guadeloupe; GRN = Grenada; GUA = Guatemala; GUF = French Guiana; HON= Honduras; JAM = Jamaica; MTQ = Martinique PAN = Panama PER = Peru; PRI = Puerto Rico; TTO = Trinidad and Tobago; URU = Uruguay; VEN = Venezuela. Country abbreviations followed by (?) represent reports without confirmed identity, which taxa were cited in literature as "aff." (affinis) or "cf." (confer) (e.g. Agrocybe aff. cylindrica).

The results obtained here are very similar to the presented in the first volume (Coimbra 2014), with Argentina and Brazil as having the highest record numbers (120 and 115, respectively). In contrast, poorly studied countries such as Honduras, Belize, Dominica and El Salvador had the lowest number of reported species. In addition, nations as Guyana, Haiti, Nicaragua, Paraguay and Suriname, as well some Caribbean Islands had no records (Figure 2). Among all publications, noteworthy are the regional contributions of Spegazzini $(1887 \mathrm{~b}, 1889,1909,1926 \mathrm{~b})$ and Raithelhuber (1974, 1977, 1988, 1991, 2004) for Argentina; Singer (1969) for Southern Argentina and Chile; and Rick (1906, 1907, 1908, 1919, 1928, 1930, 1938, 1939, 1961), Cortez, Silva and colleagues (Cortez \& Silveira 2007b, 2008; Silva et al. 2006) for Southern Brazil.

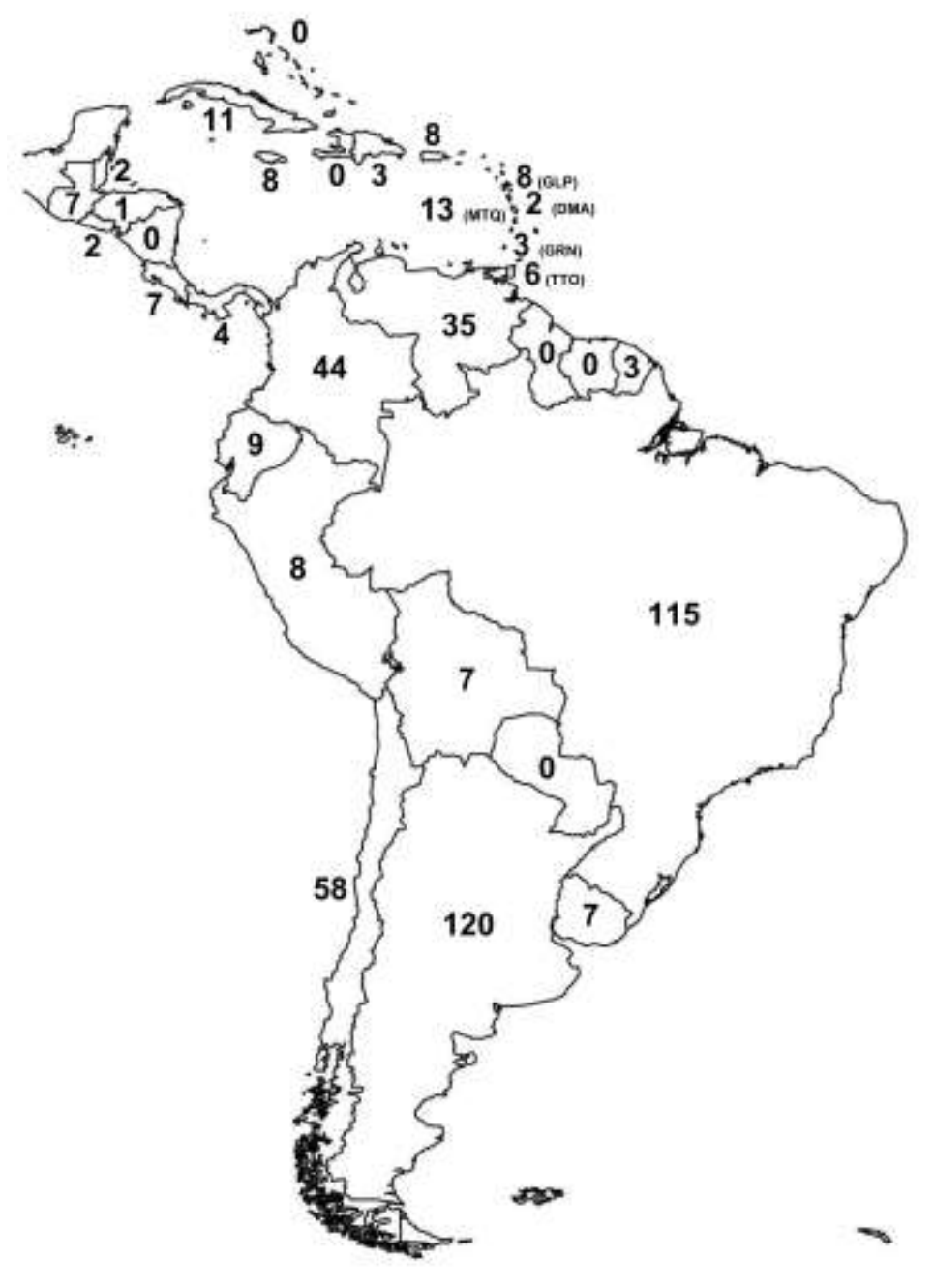

Fig. 2 - Number of Strophariaceae species recorded per country. 
Based on the number of species (1316 spp.) provided by Kirk et al. (2008), the Strophariaceae taxa reported for Central and South Americas roughly represents $\pm 22 \%$ of this family's worldwide richness. Clearly, these numbers does not reflect the real diversity of this large and heterogeneous region, highlighting the need for more specific studies.

\section{Acknowledgements}

The author thanks FACEPE (IBPG-0827-2.03/12) for PhD scholarship.

\section{References}

Albuquerque MP, Carvalho Júnior AA, Pereira AB. 2007 - Novas ocorrências de Agaricales (Basidiomycota) para o Brasil. Revista Brasileira de Biociências 5(2), 1143-1145.

Batista AC, Bezerra JL. 1960 - Basidiomycetes vulgares em o Nordeste Brasileiro. Publicações do Instituto de Micologia da Universidade do Recife 294, 1-30.

Boa E. 2004 - Wild edible fungi. A global overview of their use and importance to people. FAO, Rome.

Bononi VL, Mucci ESF, Yokomizo NKS, Guzmán G. 1984 - Agaricales (Basidiomycetes) do Parque Estadual de Campos do Jordão, SP, Brasil. Rickia 11, 85-89.

Bononi VLR, Oliveira AKM, Quevedo JR, Gugliotta AM. 2008 - Fungos macroscópicos do Pantanal do Rio Negro, Mato Grosso do Sul, Brasil. Hoehnea 35(4), 489-511.

Borgen T, Elborne SA, Knudsen H. 2006 - A checklist of the Greenland basidiomycetes. In: Boertmann D, Knudsen H. Arctic and Alpine Mycology 6. Meddelelser om Grønland Bioscience 56. The Commission for Scientific Research in Greenland, Copenhagen, 37-59.

Capelari M, Maziero R. 1988 - Fungos macroscópicos do estado de Rondônia regiões dos rios Jaru e Ji-Paraná. Hoehnea 15, 28-36.

Coimbra VRM 2014 - Checklist of Central and South American Agaricales (Basidiomycota) I: Entolomataceae. Mycosphere 5(3), 475-487, Doi 10.5943/mycosphere/5/3/10.

Cortez VG. 2008 - Type studies on South American Strophariaceae: 1. Pholiota varzeae from the Brazilian Amazon. Mycotaxon 103, 137-140.

Cortez VG, Coelho G. 2003 - Pholiota spumosa (Fr.) Singer (Strophariaceae, Basidiomycota): first record from Rio Grande do Sul, Brazil. Biociências 11(2), 133-136.

Cortez VG, Coelho G. 2004 - The Stropharioideae (Strophariaceae, Agaricales) of Santa Maria, Rio Grande do Sul, Brazil. Mycotaxon 89, 355-378.

Cortez VG, Coelho G. 2008 - Occurrence of the rare agaric Stropharia melanosperma (Strophariaceae) in Brazil. Boletín de la Sociedad Micologica de Madrid 32, 31-37.

Cortez VG, Silveira RMB. 2005 - Firts report of Agrocybe retigera (Speg.) Singer (Bolbitiaceae, Agaricales) from Brazil. Biociências 13(2), 227-229.

Cortez VG, Silveira RMB. 2007a - A new species of Stropharia with hymenial acanthocytes. Mycologia 99, 135-138.

Cortez VG, Silveira RMB. 2007b - Species of Hypholoma (Fr.) P. Kumm. (Strophariaceae, Agaricales) in Rio Grande do Sul State, Brazil. Acta Botanica Brasilica 21, 609-621.

Cortez VG, Silveira RMB. 2008 - The agaric genus Stropharia (Strophariaceae, Agaricales) in Rio Grande do Sul State, Brazil. Fungal Diversity 32, 31-57.

Courtecuisse R, Samuels GJ, Hoff M, Rossman AY, Cremers G, Huhndorf SM, Stephenson SL. 1996 - Check-list of fungi from French Guiana. Mycotaxon 107, 1-85.

Delgado AE, Urdaneta LM. 2002 - Hongos Basidiomycota, orden Agaricales, en cinco municipios del estado Zulia, Venezuela. Revista de la Facultad de Agronomía 19, 56-70.

Dennis RWG. 1961 - Fungi Venezuelani. IV. Kew Bulletin 15, 67-156.

Dennis RWG. 1968 - Agaricales from the Blue Mountains of Jamaica. Kew Bulletin 22, 73-85.

Dennis RWG. 1970 - Fungus flora of Venezuela and adjacent countries. Kew Bulletin Additional Series 3, 1-531. 
Flores Arzú R, Comandini O, Rinaldi AC. 2012 - A preliminary checklist of macrofungi of Guatemala, with notes on edibility and traditional knowledge. Mycosphere 3(1), 1-21, Doi 10.5943/mycosphere/3/1/1.

Franco-Molano AE, Corrales A, Vasco-Palacios AM. 2010 - Macrohongos de Colombia II. Listado de espécies de lós ordenes Agaricales, Boletales, Cantharellales y Russulales (Agaricomycetes, Basidiomycota). Actual Biología 32, 89-114.

Franco-Molano AE, Uribe-Calle E. 2000 - Hongos Agaricales y Boletales de Colombia. Biota Colombiana 1(1), 25-43.

Gamundi I, Horak E. 2002 - Hongos de los bosques Andino-patagónicos. Guia para el reconocimientode las espécies más comunes y atractivas. Vazquez Mazzini Editores, Buenos Aires.

George-Nascimento GMF. 2007 - Fungi Austral: guía de campo de los hongos más vistosos de Chile. Editora Corporación Chilena de la Madera, Concepción.

Grandi RAP, Guzmán G, Bononi VLR. 1984 - Adições às Agaricales (Basidiomycetes) do Parque Estadual das Fontes do Ipiranga, São Paulo, Brasil. Rickia 11, 27-33.

Guzmán G. 1978 - The species of Psilocybe known from Central and South America. Mycotaxon 7, 225-255.

Guzmán G. 1983 - The genus Psilocybe. Beihefte zur Nova Hedwigia 74. J. Cramer, Vaduz.

Guzmán G. 1995 - Supplement to the monograph of the genus Psilocybe. In: Petrini O, Horak E, eds. Taxonomic Monographs of Agari-cales. Bibliotheca Mycologica 159, 91-141.

Guzmán G. 2004 - Revision of the classification of the genus Psilocybe I. Section Neocaledonicae, a new section in Psilocybe. Revista Mexicana de Micología 18, 27-29.

Guzmán G. 2012 - New taxonomical and ethnomycological observations on Psilocybe s.s. (Fungi, Basidiomycota, Agaricomycetidae, Agaricales, Strophariaceae) from Mexico, Africa and Spain. Acta Botánica Mexicana 100, 79-106.

Guzmán G, Horak E. 1978 - A new species of Psilocybe (belonging to the P. crobula-group) from Argentina. Mycotaxon 7(3), 521-522.

Guzmán G, Cortez VG. 2004 - The neurotropic Psilocybe (Fr.) P. Kumm. (Strophariaceae, Agaricales) in Brazil: a revision of the known species, the first record of $P$. wrightii and the synonymy of $P$. caeruleoannulata. International Journal of Medicinal Mushrooms 6, 383388.

Guzmán G, Cortez VG. 2005 - A new species of Psilocybe (Agaricales, Strophariaceae) from southern Brazil. Mycotaxon 93, 95-98.

Guzmán G, Allen JW, Gartz J. 1998 - A worldwide geographical distribution of the neurotrophic fungi, an analysis and discussion. Annali dei Musei Civici di Rovereto 14, 189-280.

Guzmán G, Bononi VL, Picolo-Grandi RA. 1984 - New species, new varieties and new records of Psilocybe from Brazil. Mycotaxon 19, 343-350.

Guzmán G, Franco-Molano AE, Ramírez-Guillén F. 2007 - New section and new species of a bluing Psilocybe (Fungi, Basidiomycotina, Agaricales) from Colombia. Revista de la Academia Colombiana de Ciencias Exactas Físicas y Naturales 31(121), 469-472.

Guzmán G, Saldarriaga Y, Pineda F, Garcia G, Velazquez LF. 1994 - New species of Psilocybe from Colombia and discussion of the known species. Mycotaxon 51, 225-235.

Guzmán G, Tapia F, Nieves-Rivera ÁM, Betancourt C. 1997 - Two new bluing species of Psilocybe from Puerto Rico. Mycotaxon 63(1), 377-382.

Guzmán G, Tapia F, Ramírez-Guillén F, Baroni TJ, Lodge DJ, Cantrell SA. 2003 - New species of Psilocybe in the Caribbean, with an emendation of P. guilartensis. Mycologia 95(6), 11711180.

Guzmán G, Torres M, Ramírez-Guillén F, Ríos-Hurtado A. 2004a - Introducción al conocimiento de los Macromicetos del Chocó, Colombia. Revista Mexicana de Micología 19, 33-43.

Guzmán G, Ramirez-Guillen F, Torres M. 2004b - The hallucinogenic species of Psilocybe (Fr.) P. Kumm, (Agaricomycetidae) in Colombia, their use, new records, and new species. International Journal of Medicinal Mushrooms 6(1), 83-100. 
Halling RE, Mueller GM. 2005 - Common mushrooms of the Talamanca Mountains, Costa Rica. The New York Botanical Garden, New York.

Horak E. 1967 - Fungi Austroamericani IV. Revisión de los hongos superiores de Tierra del Fuego o Patagonia en el Herbario de C. Spegazzini en La Plata. Darwiniana 14, 355-385.

Horak E. 1980 - Fungi Basidiomycetes. Agaricales y Gasteromycetes secotioides. Flora Criptogamica de Tierra del Fuego 11(6), 1-528.

Kirk PM, Cannon PF, Minter DW, Stalpers JA. 2008 - Ainsworth \& Bisby's Dictionary of the Fungi. $10^{\text {th }}$ ed. CAB International, Wallingford.

Laessoe T, Petersen JH. 2008 - Svampelivet på ækvator. Svampe 58, 1-52.

Lechner BE, Wright JE, Popoff O. 2006 - New taxa and new records for Argentina of fungi from Iguazú National Park, Misiones. Fungal Diversity 21, 131-139.

López-Quintero C, Vasco-Palacios AM, Franco-Molano AE. 2011 - Nuevos registros de macromicetes de Colombia I. Macromicetos recolectados en zonas urbanas de Medellín (Antioquia). Actualidades Biológicas 33, 261-274.

Marcano V, Morales A, Castellano F, Salazar FJ, Martínez L. 1994 - Occurrence of psilocybin and psilocin in Psilocybe pseudobullacea (Petch) Pegler from the Venezuelan Andes. Journal of Ethnopharmacology 43, 157-159.

Matheny PB, Moreau P-A, Vizzini A, Harrower E, De Haan A, Contu M, Curti M. 2014 Crassisporium and Romagnesiella: two new genera of dark-spored Agaricales. Systematics and Biodiversity 13(1), 1-14, doi: 10.1080/14772000.2014.967823.

Meijer AAR. 2001 - Mycological work in the Brazilian state of Paraná. Nova Hedwigia 72, 105159.

Meijer AAR. 2006 - Preliminary list of the Macromycetes from the Brazilian state of Paraná. Boletim do Museu Botânico Municipal 68, 01-55.

Montagne JPFC. 1856 - Septième centurie de plantes cellulaires nouvelles, tant indigènes qu' exotiques. Annales des Sciences Naturélles, Botanique Série 4, 333-374.

Moreno G, Albertó E. 1996 - Agaricales sensu lato de Argentina I. Cryptogamie Mycologie 17(2), 61-84.

Moser MM. 2000 - Beobachtungen zur Gattung Pachylepyrium Singer. Hoppea Denkschrift der Regensburgischen Naturforschenden Gesellschaft 61, 267-274.

Moyano AJ, Daniele GM. 2003 - Nuevo registro de Psilocybe (Fungi, Basidiomycotina, Agaricales) en Argentina. Acta Botanica Mexicana 64, 25-29.

Navarro A, Betancourt C. 1992 - Hongos alucinógenos en el suroeste de Puerto Rico. International Journal of Mycology and Lichenology 5, 175-194.

Niveiro N, Albertó EO. 2012 - Checklist of the Argentine Agaricales 2. Coprinaceae and Strophariaceae. Mycotaxon 120: 505, http://dx.doi.org/10.5248/119.505.

Niveiro N, Popoff OF, Lechner BE, Albertó EO. 2014 - Pholiota oblita, new species in sect. Adiposae stirps Subflammans (Strophariaceae, Agaricomycetes), from the Argentinean Yungas. Phytotaxa 167(3), 276-282, http://dx.doi.org/10.11646/phytotaxa.167.3.6

Ovrebo CL. 1996 - The agaric flora (Agaricales) of La Selva Biological Station, Costa Rica. Revista de Biología Tropical 44(4), 39-57.

Pegler DN, Spooner BM, Lewis Smith RI. 1980 - Higher Fungi of Antarctica, the Subantarctic Zone and Falkland Islands. Kew Bulletin 35(3), 499-562.

Pegler DN. 1983 - Agaric Flora of Lesser Antilles. Kew Bulletin Additional Series 9, 1-668.

Pegler DN. 1987a - A Revision of the Agaricales of Cuba 1. Species Described by Berkeley \& Curtis. Kew Bulletin 42(3), 501-585.

Pegler DN. 1987b - A Revision of the Agaricales of Cuba 2. Species Described by Earle and Murrill. Kew Bulletin 42(4), 855-888.

Pegler DN. 1988 - A Revision of the Agaricales of Cuba 3. Keys to Families, Genera and Species. Kew Bulletin 43(1), 53-75.

Pegler DN. 1997 - The Agarics of São Paulo. An account of the agaricoid fungi (Holobasidiomycetes) of São Paulo State, Brazil. Royal Botanic Gardens Kew, London. 
Pereira AB, Putzke J. 1990 - Famílias e gêneros de fungos Agaricales (cogumelos) no Rio Grande do Sul. Livraria e Editora da FISC, Santa Cruz do Sul.

Piepenbring M. 2008 - Reportes nuevos de Agaricales para Panamá. Acta Biologica Panamensis 1, $22-38$.

Raithelhuber J. 1974 - Hongos Argentinos I. Compañia Impresora Argentina, Buenos Aires.

Raithelhuber J. 1977 - Hongos Argentinos II. Compañia Impresora Argentina, Buenos Aires.

Raithelhuber J. 1988 - Flora mycologica Argentina. Hongos II. Mycosur, Stuttgart.

Raithelhuber J. 1991 - Flora mycologica Argentina. Hongos III. Mycosur, Stuttgart.

Raithelhuber J. 2000 - Hongos Agaricales de la Isla Martin Garcia. Metrodiana 5, 1-59.

Raithelhuber J. 2004 - Nueva flora micológica Argentina. Mycosur, Stuttgart.

Reid DA, Pegler DN, Spooner BM. 1980 - An Annotated List of the Fungi of the Galapagos Islands. Kew Bulletin 35(4), 847-892.

Rick J. 1906 - Pilze aus Rio Grande do Sul. Brotéria série Botânica 5, 5-53.

Rick J. 1907 - Contribution ad monographiam Agaricacinarum Brasiliensium. Brotéria série Botânica 6, 65-92.

Rick J. 1908 - Fungi Austro-Americani IX-X. Annales Micologici 6(2), 105-108.

Rick J. 1919 - Contributio II ad Monographiam Agaricinarum Brasiliensium. Brotéria série Botânica 17, 101-111.

Rick J. 1928 - Resumo Micológico. Egatea 13, 432-439.

Rick J. 1930 - Contributio IV ad Monographiam Agaricinarum Brasiliensium. Brotéria série Botânica 24, 27-118.

Rick J. 1938 - Agarici Riograndenses. Lilloa 2, 251-316.

Rick J. 1939 - Agarici Riograndenses. IV. Lilloa 4, 75-104.

Rick J. 1961 - Basidiomycetes Eubasidii in Rio Grande do Sul - Brasília 5. Agaricaceae. Iheringia série Botânica 8, 296-450.

Rodríguez-Gallart CA. 1989 - Estudios en los macromicetos de la República Dominicana, I. Moscosoa 5, 141-153.

Sáenz JA, Macaya-Lizano AV, Nassar M. 1983 - Hongos comestibles, venenosos y alucinatorios de Costa Rica. Revista de Biología Tropical 31, 201-207.

Silva PS, Cortez VG, Silveira RMB. 2006 - The mycobiota of Itapuã Park, Rio Grande do Sul, Brazil.I. Species of Strophariaceae (Agaricales). Mycotaxon 97, 219-229.

Silva PS, Cortez VG, Silveira RMB. 2008 - Strophariaceae (Agaricales, Basidiomycota) no Parque Estadual de Itapuã, Viamão, Rio Grande do Sul: chave para identificação das espécies. Revista Brasileira de Biociências 6(3), 253-258.

Silva PS, Cortez VG, Silveira RMB. 2009 - New species of Stropharia from Araucaria angustifolia forests of southern Brazil. Mycologia 101(4), 539-544. doi: 10.3852/08-097

Silva PS, Cortez VG, Silveira RMB. 2012 - Synopsis of the Strophariaceae (Basidiomycota, Agaricales) from Floresta Nacional de São Francisco de Paula, Rio Grande do Sul State, Brazil. Hoehnea 39(3), 479-487.

Silva PS, Guzmán G, Cortez VG, Ramírez-Guillén F, Silveira RMB. 2007 - Psilocybe subbrunneocystidiata (Strophariaceae, Agaricales): a new species from southern Brazil. Mycotaxon 102, 203-207.

Silva PS, Ramírez-Cruz V, Cortés-Pérez A, Guzmán G, Guzmán-Dávalos L, Silveira RMB. 2013 Deconica neorhombispora (Agaricales, Strophariaceae): new combination and synonym. Sydowia. 65(2), 321-328.

Silva PS, Silveira RMB, Cortez VG. 2014 - Notes on Deconica and Psilocybe (Agaricales) from the southern border of Atlantic forest and Pampa biomes of Brazil. Sydowia 66(2), 217228.

Singer R. 1950 - Type studies on Basidiomycetes IV. Lilloa 23, 147-246.

Singer R. 1953 - Type studies on Basidiomycetes. VI. Lilloa 26, 57-159.

Singer R. 1955 - New and interesting species of Basidiomycetes. IV. Mycologia 47, 763-777.

Singer R. 1959 - New and interesting species of Basidiomycetes. VII. Mycologia 51, 578-594. 
Singer R. 1965 - Interesting and new Agaricales from Brazil. Atlas do IMUR 2, 15-59.

Singer R. 1969 - Mycoflora australis. Beihefte zur Nova Hedwigia 29, 1-405.

Singer R. 1973 - Diagnoses Fungorum Novorum Agaricalium III. Beiheft zur Sydowia 7, 1-106.

Singer R. 1978 - Interesting and new species of Basidiomycetes from Ecuador II. Nova Hedwigia 29, 1-98.

Singer R. 1986 - The Agaricales in modern taxonomy. $4^{\text {th }}$ ed. Koeltz Scientific Books, Koenigstein.

Singer R. 1989 - New taxa and new combinations of Agaricales (Diagnoses Fungorum Novorum Agaricalium IV). Fieldiana 21, 1-133.

Singer R, Digilio APL. 1951 - Pródromo de la Flora Agaricina Argentina. Lilloa 25, 5-461.

Sobestiansky G. 2005 - Contribution to a Macromycete Survey of the States of Rio Grande do Sul and Santa Catarina in Brazil. Brazilian Archives of Biology and Technology 48, 437-457.

Sotão HMP, Bononi VLR, Figueiredo TS. 1991 - Basidiomycetes de manguezais da Ilha de Maracá, Amapá, Brasil. Boletim do Museu Paraense Emílio Goeldi série Botânica 7(1), 109-114.

Spegazzini C. 1887a - Fungi patagonici. Boletín de la Academia Nacional de Ciencias en Córdoba, Republica Argentina 11, 5.

Spegazzini C. 1887b - Fungi fuegiani. Boletín de la Academia Nacional de Ciencias en Córdoba, Republica Argentina 11, 135-308.

Spegazzini C. 1899 - Fungi Argentini novi vel critici. Anales del Museo Nacional de Historia Natural Buenos Aires 6, 6-365.

Spegazzini C. 1909 - Mycetes argentinensis IV. Anales del Museo Nacional de Historia Natural Buenos Aires 19, 257-458.

Spegazzini C. 1922 - Cryptogamae nonnullae fuegianae. Anales de la Sociedad Cientifica Argentina 94, 59-85.

Spegazzini C. 1925 - Relación de un paseo hasta el Cabo de Hornos. Boletin de la Academia Nacional de Ciencias (Córdoba) 27, 321-404.

Spegazzini C. 1926a - Contribución al conocimiento de la flora micológica de las Sierras de Córdoba. Boletín de la Academia Nacional de Ciencias en Córdoba 29, 113-190.

Spegazzini C. 1926b - Observaciones y adiciones a la Micologia Argentina. Boletín de la Academia Nacional de Ciencias en Córdoba 28, 267-423

Stijve T, Meijer AAR. 1993 - Macromycetes from the State of Paraná, Brazil 4: The psychoactive species. Arquivos de Biologia e Tecnologia 36, 313-329.

Theissen SJF. 1912 - Hymenomycetes Riograndenses. Brotéria 10, 1-24.

Valenzuela E, Moreno G, Grinbergs J. 1992 - Agaricales sensu lato de Chile. I. Boletín de la Sociedad Micológica de Madrid 17, 81-93.

Velázquez LF, Saldarriaga Y, Pineda F, Gracia G. 1991 - Nuevos registros de hongos en Colombia (Departamento de Antioquia). Descripción de algunos Agaricales. Actualidades Biológicas 18(66), 74-94.

Wartchow F, Carvalho AS, Sousa MCA, Cortez VG. 2007 - Some coprophilous Psilocybe (Strophariaceae) from Pernambuco State, Northeast Brazil. Sitientibus Série Ciências Biológicas 7(2), 150-153.

Watling R. 1992 - Observations on the Bolbitiaceae- 30. Some Brazilian taxa. Boletín de la Sociedad Argentina de Botánica 28, 77-103.

Wright JE, Albertó E. 2002 - Guía de los Hongos de La Región Pampeana. I. Hongos con Laminillas. L.O.L.A., Buenos Aires.

Wright JE, Lechner BE, Popoff O. 2008 - Hongos. Atlas pictórico del Parque Nacional Iguazú. L.O.L.A., Buenos Aires.

Yokoyama K. 1987. The coprophilous species of Psilocybe from Peru. In: Inove H, eds. Studies on Cryptogams in Southern Peru. Tokai Univ. Press, Tokyo, 145-149. 